\title{
Guided wave excitation by a CLoVER transducer for structural health monitoring: theory and experiments
}

\author{
K I Salas and C E S Cesnik ${ }^{1}$ \\ Department of Aerospace Engineering, The University of Michigan, 1320 Beal Avenue, \\ Ann Arbor, MI 48109, USA \\ E-mail: ksalas@umich.edu and cesnik@umich.edu
}

Received 21 October 2008, in final form 28 April 2009

Published 3 June 2009

Online at stacks.iop.org/SMS/18/075005

\begin{abstract}
The guided wave (GW) field excited by a wedge-shaped, anisotropic piezocomposite transducer, surface-bonded on an isotropic substrate is investigated with applications to large area structural health monitoring. This investigation supports the development of the composite long-range variable-direction emitting radar (CLoVER) transducer. The analysis is based on the three-dimensional equations of elasticity, and the solution yields expressions for the field variables that are able to capture the multimodal nature of GWs. The assumption of uncoupled dynamics between the actuator and substrate is used, and their interaction is modeled through shear tractions along the transducer's radial edges. A similar problem is modeled using three-dimensional finite element simulations to assess the spatial and transient accuracy of the solution. Experimental tests are also conducted on pristine structures to validate the accuracy of the theoretical approach. The experimental studies employ CLoVER transducers developed in-house, and their manufacturing procedure is briefly described. Frequency response experiments based on piezoelectric sensors are conducted to assess the performance of the solution in the frequency domain. These tests are complemented by laser vibrometer measurements that allow the spatial and temporal evolution of the solution to be evaluated. The numerical simulations and experimental tests show that the wave time of arrival, radial attenuation, and azimuthal distribution are well captured by the theoretical solution.
\end{abstract}

(Some figures in this article are in colour only in the electronic version)

\section{Nomenclature}

$A$
$A^{*}$
$b$
$\bar{b}$
$C$
$c_{p}, c_{s}$
$c_{k}$
$D$
$d_{i j}$
$E$
$\overline{\mathbf{F}}$

Area

Aspect ratio

Substrate half-thickness

Residue

\section{Capacitance}

$c_{p}, c_{s} \quad$ Dilatational/shear wave speed in isotropic material

$c_{k} \quad$ Complex Fourier coefficients of shear tractions

$D \quad$ Dispersion equation for Rayleigh-Lamb waves

$d_{i j} \quad$ Piezoelectric coupling coefficient $(i, j=1-3)$

$E \quad$ Young's modulus for an isotropic material

F Shear stresses Fourier transform vector

1 Author to whom any correspondence should be addressed. $g_{i j} \quad$ Piezoelectric constants $(i, j=1-3)$

$\mathbf{H}, \varphi \quad$ Helmholtz displacement components

$h, w$ Generic functions

$\tilde{H}_{k} \quad$ Hankel transform of order $k$

$J_{m} \quad$ Bessel function of the first kind and order $m$

$k \quad$ Dielectric constant

$n \quad$ Number of half-cycles in toneburst signal

$p \quad$ Capacitance function

$r, \theta \quad$ Radial/azimuthal position

$r^{*} \quad$ Non-dimensional sensor dimension

$\Delta r \quad$ Radial dimension

$R \quad$ Radius 
$R_{\mathrm{O}} \quad$ Transducer's outer radius

$R_{\mathrm{I}} \quad$ Transducer's inner radius

$s(t) \quad$ Toneburst signal

$S(\omega) \quad$ Fourier transform of toneburst signal

$t \quad$ Time

$\bar{t} \quad$ Thickness

$u(\cdot) \quad$ Unit step function

u Displacement vector

$V \quad$ Voltage

$w^{*} \quad$ Non-dimensional sensor width

$x, y, z \quad$ Cartesian coordinates

$\hat{z} \quad$ Arbitrary complex variable

$\Gamma, \Psi$ Coefficient matrices used in the theoretical solution

$\delta(\cdot) \quad$ Dirac delta function

$\Delta \theta \quad$ Azimuthal dimension

$\epsilon_{i j} \quad$ Strain components $(i, j=x, y$ or $r, \theta)$

$\epsilon_{0} \quad$ Permittivity constant

$\varepsilon \quad$ Error between function and Fourier series representation

$\theta^{*} \quad$ Non-dimensional sensor azimuthal span

$\theta_{\mathrm{L}} \quad$ Transducer's left azimuthal edge

$\theta_{\mathrm{R}} \quad$ Transducer's right azimuthal edge

$\lambda, \mu \quad$ Substrate Lamé constants

$v \quad$ Poisson's ratio for an isotropic material

$\xi \quad$ Radial wavenumber

$\xi_{x}, \xi_{y} \quad$ Wavenumber along $x$ - and $y$-directions

$\rho \quad$ Substrate material density

$\tau_{0} \quad$ Traction amplitude

$\sigma \quad$ Stress tensor

$\phi \quad$ Azimuthal wavenumber

$\omega \quad$ Angular frequency

$\omega_{0} \quad$ Center frequency of toneburst signal

\section{Subscript}

a Actuator

A Antisymmetric mode

B Substrate

e Element property

$P \quad$ Piezoelectric

s Sensor

S Symmetric mode

uc Unit cell

\section{Introduction and background}

The objective of structural health monitoring (SHM) is to obtain real-time information about the condition of a structural component. This involves interrogating the structure of interest using an on-board network of transducers to determine whether any damage is present, and if so its location, type, and severity. It is envisioned that the information obtained from these systems could be used as input to damage prognosis algorithms which would make a prediction about the remaining useful life of the component based on its current condition. There are multiple safety and cost-saving benefits associated with the implementation of this technology in aerospace, mechanical, and civil structures. An introduction to this field was presented in the work by Farrar et al [1], where the process of SHM was defined in terms of a four-step pattern recognition process. These steps dealt with evaluation of the operational environment of the SHM system, the acquisition and interpretation of transducer data, and the development of statistical models for feature discrimination. Similarly, the work of Worden et al [2] provided a set of axioms for the development of SHM methodologies where key requirements and constraints were discussed.

Guided wave (GW) testing methods have gained importance in SHM applications primarily because of their ability to be transmitted over long distances over the surface as well as through the thickness of a structure with little attenuation [3]. In addition, their active nature allows them to be used for inspection on demand. Another important benefit of this approach is the sensitivity of different GW modes to a variety of structural defects. For instance, the fundamental symmetric $\left(\mathrm{S}_{0}\right)$ Lamb mode is sensitive to through-the-thickness damage due to its dominant in-plane components, and therefore is well suited for the detection of, for example, full- or part-depth holes. Similarly, the $\mathrm{A}_{0}$ mode is better suited for surface damage (e.g., surface cracks) due to its dominant out-of-plane component. A comprehensive review of this SHM approach was presented by Raghavan and Cesnik [3].

The following subsections provide a brief background on the key aspects that are addressed in this paper. Previous efforts on GW modeling are discussed first, followed by a brief overview of the available technology for GW transduction. The different testing approaches available for GW experiments are then discussed. These components are combined in the last subsection where the scope of the present investigation is described.

\subsection{Analysis of $G W$ propagation in isotropic plates}

After the early development of Lamb wave theory (e.g., the works of Lamb [4] and Gazis [5]), there has been much effort in elastodynamics research to develop solutions for the GW field excited by surface-bonded transducers. Various approaches have been taken towards this goal and some of these are highlighted in this section. Several researchers have analyzed the propagation of GWs in isotropic plates by modeling the actuator as a combination of point sources. For example, Wilcox et al [6] used the Huygens principle to model the acoustic field induced by interdigital polyvinylidene fluoride (PVDF) transducers. In that approach, the electrode fingers were divided into individual elements, each of which was modeled as a point source causing a normal traction on the surface of the substrate. Each of these sources was represented through an excitability function and radiation pattern, both of which depended on wavenumbers found from the RayleighLamb dispersion relation. The contribution from the actuator was then found by integrating the contributions from each individual point. The predictions from this analysis were shown to compare well with experimental measurements for rectangular and wedge-shaped transducers. Along a similar direction, although in a more general framework, Achenbach et al [7] expressed the displacements induced by a timeharmonic point load of arbitrary direction, applied either 
internally or on the surface, as an expansion of symmetric and antisymmetric Lamb modes using Hankel functions as the carrier waves. In their approach, the principle of elastodynamic reciprocity was used to find the expansion coefficients for each mode.

Different studies have used reduced order plate theories in the analysis of wave propagation in an effort to obtain more computationally efficient, but significantly simplified solutions. For instance, Lin and Yuan [8] modeled the excitation of GWs on isotropic substrates using Mindlin plate theory accounting for transverse shear and rotary inertia effects, and considering only axially symmetric motions. The effect of the transducer on the plate was modeled as uniform bending moments around the edges of the actuator. In an effort to correlate their analysis with experimental results, a model for the sensor response under the excited GW field was developed. The experimental measurements, which employed piezoceramic disks, displayed reasonable agreement with the theory until frequency-thickness products of $160 \mathrm{kHz} \mathrm{mm}$, as the shear correction factor needed in the formulation was chosen to match one dispersion curve corresponding to the 3D elasticity solution. Similarly, Rose and Wang [9] used Mindlin plate theory to derive source solutions that could be combined to represent finite-dimensional transducers. They argued that the range of validity of their solution made it applicable to typical aerospace structures. A higher-order plate theory, considering first-order normal strains and second-order transverse shear strains, was developed by Yang and Yuan [10]. In their work, it was argued that this theory produced good agreement with 3D elasticity solutions until the first cut-off frequencies using a more efficient approach. However, the main limitation with this type of approaches is that they are limited to low frequency-thickness products and, in most cases, can only model the fundamental antisymmetric mode. As was previously mentioned, one of the main advantages of using GWs is their sensitivity to different damage types, which can be better exploited at higher frequency-thickness products. Furthermore, while damage detection can be typically achieved using the fundamental modes, it may be necessary to use higher modes if the type of defect is to be identified. In this case, only 3D elasticity models will be reliable.

A more accurate model was developed by Giurgiutiu, where the GW field excited by an infinitely wide piezoelectric actuator and an isotropic substrate was modeled using 2D elasticity [11, 12]. The interaction between both parts was modeled as shear tractions along the transducer edges, and the solution was found using Fourier transforms and the residue theorem from complex calculus. The concept of Lamb wave tuning was demonstrated and good agreement with experiments was reported. Following this approach, Raghavan and Cesnik developed 3D elasticity solutions for GW excitation by finite-dimensional transducers in isotropic and composite structures [13-15]. The analysis used Fourier transforms and complex calculus, and a rigorous Fourier inversion procedure was presented. Several transducer constructions (piezoelectric wafers and piezocomposite transducers) and geometries (rectangular, circular, and ring-shaped) as well as structural configurations (pipes and plates) were considered, and the solution was used to determine optimal actuator and sensor dimensions for use in SHM systems.

Different approaches to numerically model the propagation of GW have also been proposed. Typical solution methodologies, such as the finite-difference and finite element methods, are not ideally suited for GW analysis due to their high computational cost. An alternative approach was proposed by Lee and Staszewski [16] who applied the local interaction simulation approach (LISA) to analyze the GW propagation in metallic structures. Following this approach, the domain was divided into elementary cells that were considered to be discontinuous among each other. Therefore, displacement continuity was enforced at each cell node. The results from this method were favorably correlated with experimental data, and subsequently used in modeling the interaction of GWs with different structural defects $[16,17]$.

\subsection{Transducers for $G W$ excitation in SHM}

Several transducer options are available for the excitation of GWs, and a brief summary of these is presented in this section. For a comprehensive review of this area, the reader is referred to the work of Raghavan and Cesnik [3]. The GW field is usually excited using piezoelectric transducers, with the most common transducer type being simple piezoelectric wafers (commonly referred to as piezos) bonded on the surface of the structure to be inspected. Typical materials used for the construction of these wafers include lead zirconium titanate ceramics (PZT) and polyvinylidene fluoride (PVDF) films. These wafers are thin, light, and unobtrusive and are very convenient for surface-bonded-based inspection. Among these, however, PZT is usually preferred since PVDF has a high compliance and low inverse piezoelectric effect which results in poor actuator-sensor response [3]. The main disadvantage in using these, however, is that, although thin and light, the ceramic material is also very brittle and does not have good surface conformability. This limitation is particularly important for the shell-type structures usually encountered in aerospace applications. In order to alleviate this problem, different anisotropic piezocomposite transducer (APT) concepts have been designed and manufactured in recent years. Bent and Hagood [18] designed the active fiber composite (AFC) transducer using extruded, cylindrical piezoceramic fibers embedded in an epoxy matrix. These fibers are actuated through the use of interdigitated electrode patterns printed on a copper-clad kapton film. This construction presented numerous advantages, in particular, a greatly enhanced surface conformability, high strain energy density, and focused strain actuation. The implementation of interdigitated electrodes allowed the use of the $3-3$ piezoelectric effect, where the poling of the device is such that the highest piezoelectric coupling coefficient coincides with the intended actuation direction, as shown in figure 1(a), theoretically allowing these devices to induce strains at least twice as large as those obtained with simple PZT actuators. An alternative concept, the macro-fiber composite (MFC) transducer illustrated in figure 1(b), was developed by 


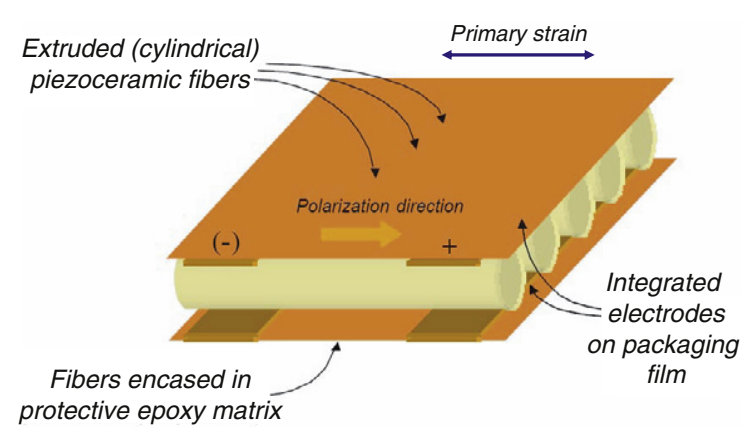

(a)

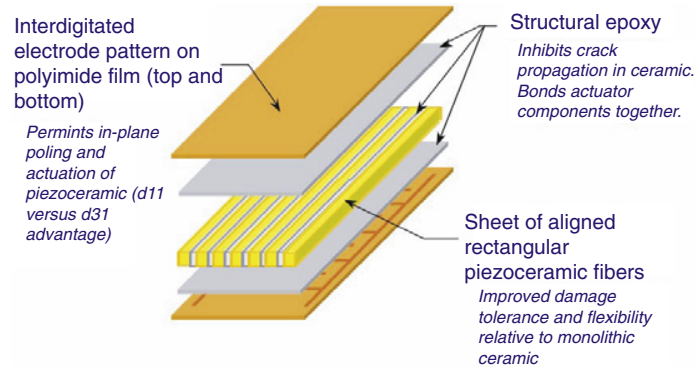

(b)

Figure 1. The two primary types of APT [19]. (a) Active fiber composite (AFC) transducer, (b) macro-fiber composite (MFC) transducer.

researchers at NASA Langley [19]. This type of construction is very similar to the AFC, except that it uses rectangular fibers obtained by dicing a piezoceramic wafer using high-precision dicing saws $[20,21]$ yielding a more repeatable manufacturing procedure. The most salient non-piezoelectric alternative is that of electromagnetic acoustic transducer arrays (EMATs) which typically require permanent magnets to be bonded on the surface of the structure under inspection (see for example [22]). These devices use electromagnetic fields to generate forces on the substrate that result in the excitation of GWs. Since these devices are typically bulky, they are not well suited for aerospace applications and will consequently not be considered in this work from this point onwards.

\subsection{GW experiments for SHM applications}

GW experiments directed towards SHM applications have been performed in the past by several researchers in both isotropic and composite structures. In general, two approaches have been used to record the GW field generated experimentally. The first consists of bonding piezoelectric sensors on the surface of the structure under investigation to record the strain field produced by the actuator. Using this approach, the sensor can be sized so that it is insensitive to specific Lamb wave modes which is a desirable feature for damage detection. An additional benefit is that there is no limit to the Lamb wave propagation frequency that can be sensed. Some important disadvantages of this approach are the facts that the sensor's performance is susceptible to environmental conditions, such as electromagnetic interference (EMI), and that information is only recorded at the point where the sensor is placed. Raghavan and Cesnik have used this approach successfully in sensing the GW field generated by piezoelectric-based actuators in pristine and damaged isotropic structures [13, 23]. More recently, this was used to study the effects of environmental conditions, such as elevated temperatures, on GW propagation and damage detection [24]. This approach has also been used for damage detection in composite structures. The works of Badcock et al [25], who used embedded piezoelectric transducers to detect impact damage in composite plates, and Kessler et al [26], where surface-bonded piezoelectric patches were used for detecting different damage types in composite laminates and sandwich panels, are typical examples of this application.
The second approach is based on the non-contact technique of laser vibrometry, where a laser beam is used to record the out-of-plane velocities induced by the piezoelectric actuator using the Doppler shifting phenomenon. An overview of the different non-contact techniques available for SHM applications can be found in the work of Staszewski et al [27]. This approach allows outstanding visualization of the wave field, which is valuable in studying its interaction with different damage types. This feature is also critical in the experimental analysis of wave propagation in composite laminates, where wave steering phenomena are typically present [15]. In spite of these advantages, this method is impractical for on-line based inspection, and post-processing of the data recorded (smoothing and denoising) is usually necessary before it can be effectively analyzed [27]. Staszewski et al have used this method to study the GW field excited by piezoelectric transducers in metallic structures, with their initial studies successfully verifying this technique's performance in sensing low frequency $(75 \mathrm{kHz}) \mathrm{GWs}$ in isotropic plates [27]. Their investigation was later directed towards detecting different damage types such as circular holes, rectangular notches, and cracks [28, 29]. More recently, they have used 3D laser vibrometry to directly measure all displacement components with applications to fatigue crack detection [30]. This approach has also been used for GW visualization in quasi-isotropic composite laminates (see for example [31]).

\subsection{Scope of the present work}

This study is concerned with the development of a 3D elasticity theory for GW excitation by a wedge-shaped APT in isotropic plate-like structures. The construction of this theory supports the development of the composite longrange variable-direction emitting radar (CLoVER) transducer presented by the authors as an alternative concept for damage interrogation in SHM systems. The theoretical framework is based on the work of Raghavan and Cesnik [13, 14], and the resulting solution is therefore able to capture the multimodal nature of GWs. The paper begins with a brief description of the CLoVER transducer where some of its salient features are briefly described. The boundary value problem of linear elasticity is then formulated and tailored to the case of a CLoVER sector. The solution process is later described in detail and the resulting displacement equations are presented. 


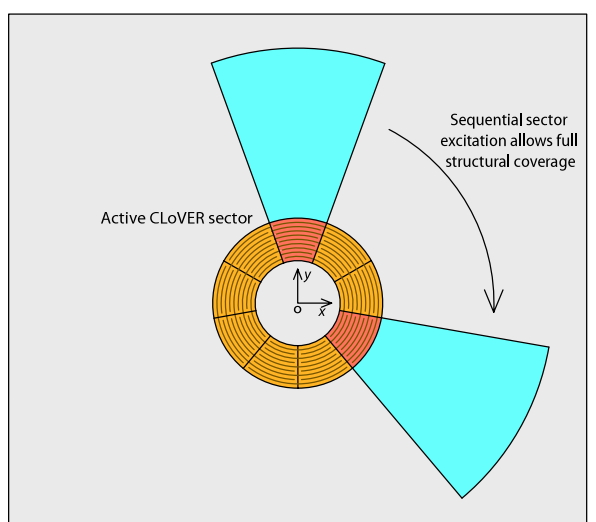

(a)

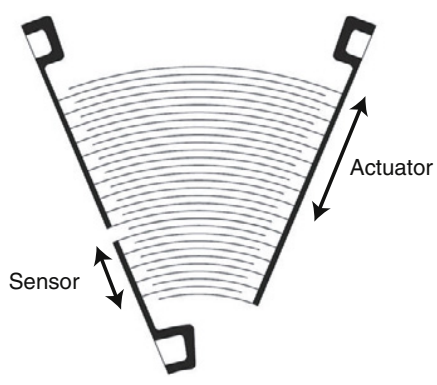

(b)

Figure 2. (a) Sequential damage interrogation approach proposed with CLoVER transducers; (b) electrode design used in first generation of CLoVER sectors.

Results from 3D finite element simulations are then used to determine the accuracy of the theoretical result as a solution to the elasticity problem considered. Subsequently, the fitness of the theoretical result in describing the actual physical system is explored through a comprehensive set of experimental tests in pristine isotropic structures. The performance of the solution in the frequency domain is verified using sensor-based experiments for both the symmetric and antisymmetric modes. These results are then complemented by laser vibrometer measurements that allow the temporal and spatial evolution of the solution to be assessed.

\section{The CLoVER transducer}

The CLoVER transducer has been introduced $[32,33]$ as an alternative concept for efficient damage interrogation and GW excitation in SHM systems. This transducer consists of a collection of wedge-shaped APTs arranged in a circular array that are individually activated in a sequential manner to scan a complete $360^{\circ}$ structural range, as shown in figure 2(a).

The geometry of a CLoVER sector allows the amplitude of the induced displacements to be larger than those for a similarly sized ring configuration for similar electric current inputs. This is related to the decreasing capacitance of the device as its azimuthal span is decreased. In addition, the interdigitated electrode design used in the first generation of CLoVER transducers uses two independent radial subdivisions that are obtained by connecting different sets of electrode fingers to independent electrode lines. This variable-length feature can be used to achieve modal selectivity, which is a desired feature in GW testing (see for instance [34]). This can be achieved by selecting the radial dimension according to the wavelength of the desired mode. Moreover, these subdivisions give each sector in the transducer the ability to independently act as an actuator and sensor, as schematically shown in figure 2(b), where it can be seen on the left-hand-side of the electrode pattern that the upper and lower sections are separate. Using the radial subdivisions for independent acting and sensing could decrease the number of separate transducers needed for inspection. Finally, the composite construction characteristic of APTs gives the CLoVER transducer the ability to conform to curved surfaces (e.g., aircraft fuselages), an increased resistance to environmental damage, and a higher specific strength than monolithic piezoelectric wafers. The advantages of the CLoVER concept are discussed in detail in separate works by the authors $[33,35]$.

\section{Boundary value problem formulation}

The GW field excited by a finite-dimensional transducer bonded on the surface of an isotropic substrate is modeled as a boundary value problem of linear elasticity. The dynamics of the actuator and the substrate are assumed to be uncoupled, and their interaction is modeled as surface tractions along the edges of the transducer. There are two implications to this assumption. First, the bonding layer between the actuator and the substrate is assumed to be infinitely thin and not shear deformable, that is, the two parts are assumed to be perfectly bonded. This ensures that strains are transferred only along the actuator edges. This assumption is clearly an idealization, as the bonding layer will have a finite thickness in actual applications. Valuable insight into this aspect was provided by the work of Crawley and de Luis [36], who considered the case of a piezoelectric actuator surface-bonded on a substrate under static conditions. They showed that, for a bonding layer with finite thickness, this assumption becomes accurate if the product of the actuator's modulus and thickness is much larger than that of the substrate on which it is bonded. In fact, it was shown that as this ratio approaches zero, the assumption becomes the exact solution. It was also shown that if this condition was not satisfied, a shear lag solution considering strain transfer along the length of the actuator was necessary. Secondly, so that the dynamics of the actuator can be ignored, its inertia must be a small fraction of the total inertia of the system in the region where strains are transferred. These assumptions are necessary if tractable semi-analytical solutions are to be obtained. Similar models have been used in the past and good agreement with experiments has been obtained [11-14]. 


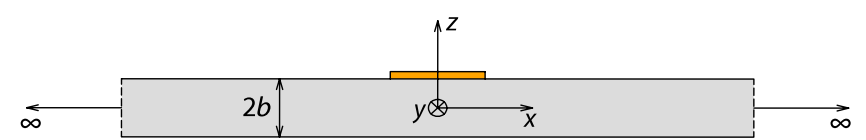

(a)

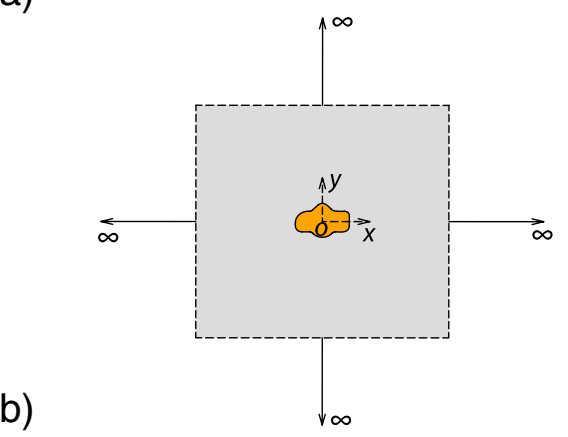

Figure 3. Transducer bonded on surface of infinite plate. (a) Cross-sectional view, (b) top view.

Based on these assumptions, consider an isotropic substrate of thickness $2 b$, with the coordinate system centered midway through its thickness, as depicted in figure 3(a). The substrate is of infinite dimensions along the $x$ - and $y$ directions, as shown in figure 3(b), and the transducer is bonded on the surface $z=b$. Using the constitutive law for isotropic materials and linear strain-displacement relations, the equilibrium equations in the absence of body forces may be expressed as:

$$
(\lambda+\mu) \nabla \nabla \mathbf{u}+\mu \nabla^{2} \mathbf{u}=\rho \ddot{\mathbf{u}} .
$$

As previously mentioned, the transducer is replaced by surface tractions on the top surface of the substrate, while the bottom surface is traction free. Therefore, the following set of boundary conditions applies:

$$
\begin{gathered}
\boldsymbol{\sigma}(x, y,-b) \cdot \mathbf{n}_{\mathbf{l}}=\mathbf{0} \\
\boldsymbol{\sigma}(x, y, b) \cdot \mathbf{n}_{\mathbf{u}}=\sigma_{0}
\end{gathered}
$$

where $\mathbf{n}_{\mathbf{l}}=\left[\begin{array}{lll}0 & 0 & -1\end{array}\right]^{\mathrm{T}}, \mathbf{n}_{\mathbf{u}}=\left[\begin{array}{lll}0 & 0 & 1\end{array}\right]^{\mathrm{T}}$ and:

$$
\boldsymbol{\sigma}_{0}=\left[\begin{array}{c}
\sigma_{x z}^{\circ} \\
\sigma_{y z}^{\circ} \\
0
\end{array}\right] \text {. }
$$

Equation (4) reflects the fact that the surface-bonded actuator only induces shear stresses on the substrate. A solution to this problem, for the general case of an arbitrarily-shaped transducer, was presented by Raghavan and Cesnik [13, 37] and is outlined below. The displacement vector, $\mathbf{u}$, is decomposed into its Helmholtz components as:

$$
\begin{gathered}
\mathbf{u}=\nabla \varphi+\nabla \times \mathbf{H} \\
\nabla \cdot \mathbf{H}=0 .
\end{gathered}
$$

The first term in equation $(5 a)$ represents the gradient of a scalar potential associated with dilatational deformations, while the second term represents the curl of a vector potential associated with constant volume distortions. This decomposition results in the following four differential equations:

$$
\begin{aligned}
\nabla^{2} \varphi & =\frac{\ddot{\varphi}}{c_{p}^{2}} \\
\nabla^{2} \mathbf{H} & =\frac{\ddot{\mathbf{H}}}{c_{s}^{2}}
\end{aligned}
$$

where $c_{p}$ and $c_{s}$ correspond to the dilatational and shear wave speeds, respectively, which are defined through:

$$
\begin{gathered}
c_{p}^{2}=\frac{\lambda+2 \mu}{\rho} \\
c_{s}^{2}=\frac{\mu}{\rho} .
\end{gathered}
$$

The solution to these equations, under harmonic excitation, is obtained using two-dimensional Fourier transforms, along with the set of boundary conditions given by equations (2) and (3). The spatial version of the two-dimensional Fourier transform, $\bar{F}\left(\xi_{x}, \xi_{y}\right)$, of a generic function, $f(x, y)$, is defined as:

$$
\bar{F}\left(\xi_{x}, \xi_{y}\right)=\int_{-\infty}^{\infty} \int_{-\infty}^{\infty} f(x, y) \mathrm{e}^{\mathrm{i}\left(\xi_{x} x+\xi_{y} y\right)} \mathrm{d} x \mathrm{~d} y .
$$

Applying the 2D spatial Fourier transform to equation (6a) results in:

$$
-\left(\xi_{x}^{2}+\xi_{y}^{2}\right) \bar{\varphi}+\frac{\partial^{2} \bar{\varphi}}{\partial z^{2}}=-\frac{\omega^{2}}{c_{p}^{2}} \bar{\varphi}
$$

where the derivatives property of the Fourier transform has been used. It can be seen that the Fourier transform application resulted in a partial differential equation being reduced to an ordinary differential equation in the thickness domain. The solution to this equation can be expressed as:

$$
\bar{\varphi}=A \sin \alpha z+B \cos \alpha z
$$

where the term $\alpha$ is defined as:

$$
\alpha \equiv \sqrt{\frac{\omega^{2}}{c_{p}^{2}}-\xi^{2}}
$$

and the radial wavenumber $\xi$ is defined through $\xi^{2}=\xi_{x}^{2}+\xi_{y}^{2}$. A similar procedure is applied to each component of the distortional field, $\mathbf{H}$, which results in the introduction of eight constants that determine the displacement field. The solution to $\bar{\varphi}$ and each component of $\overline{\mathbf{H}}$ are then substituted in the Fouriertransformed version of equation $(5 a)$ to find the displacement components in the Fourier domain. This result is subsequently combined with a linear kinematic relationship of the form given in equation (12) to obtain the strain components.

$$
\epsilon_{i j}=\frac{1}{2}\left(u_{i, j}+u_{j, i}\right) .
$$

The strain components are then used in conjunction with a linear elastic constitutive equation to determine the stress components. The boundary conditions given by equations (2) and (3) are subsequently used along with equation (5b) to form a system of equations from which the necessary constants can be found. The dispersion equation for Rayleigh-Lamb waves 


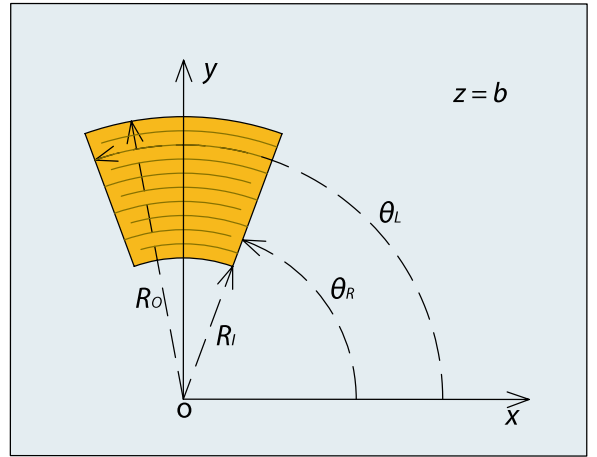

(a)

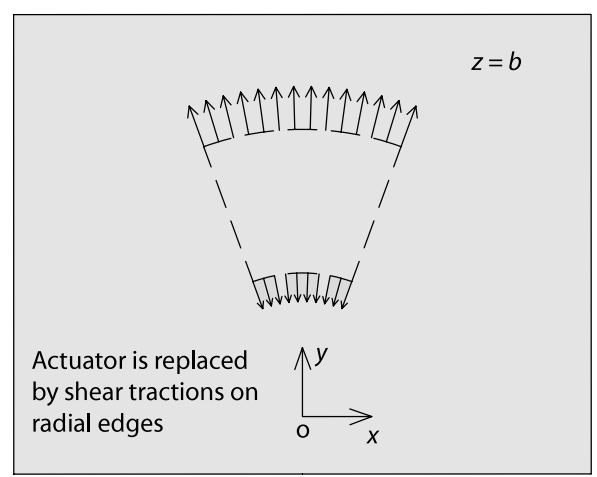

(b)

Figure 4. (a) Geometry of a CLoVER sector; (b) transducer replaced by shear tractions along radial edges.

results from seeking a non-trivial solution to this system of equations. A detailed exposition of this procedure can be found in the book by Graff [38]. The resulting displacement field can be separated into symmetric and antisymmetric components. To facilitate the presentation of the theory, only the results corresponding to the antisymmetric mode will be presented from this point on. The derivation of the symmetric component follows an analogous sequence. Note that the complete displacement field is obtained by summing the contribution from both modes. After applying the 2D inverse Fourier transform, the Cartesian displacement components expressed in Cartesian coordinates are obtained as:

$$
\begin{aligned}
& \mathbf{u}_{A}(x, y, t)=\int_{-\infty}^{\infty} \int_{-\infty}^{\infty} \frac{\Gamma_{A}\left(\xi_{x}, \xi_{y}\right)}{D_{A}(\xi)} \\
& \quad \times \Psi_{A}\left(\xi_{x}, \xi_{y}\right) \cdot \overline{\mathbf{F}}\left(\xi_{x}, \xi_{y}\right) \mathrm{e}^{-\mathrm{i}\left(\xi_{x} x+\xi_{y} y-\omega t\right)} \mathrm{d} \xi_{x} \mathrm{~d} \xi_{y} .
\end{aligned}
$$

Note that equation (13) provides the displacement components at the surface $z=b$. All subsequent analytical expressions presented here will be for this surface as well. In equation (13), $\boldsymbol{\Gamma}_{A}$ and $\boldsymbol{\Psi}_{A}$ are matrices of coefficients, $\overline{\mathbf{F}}$ is a vector containing the Fourier transform of the surface tractions, and $D_{\mathrm{A}}$ corresponds to the dispersion relation for the antisymmetric mode of Rayleigh-Lamb waves given by:

$$
D_{\mathrm{A}}=\left(\xi^{2}-\beta^{2}\right)^{2} \sin \alpha b \cos \beta b+4 \xi^{2} \alpha \beta \cos \alpha b \sin \beta b
$$

where the term $\beta$ is defined as:

$$
\beta \equiv \sqrt{\frac{\omega^{2}}{c_{s}^{2}}-\xi^{2}}
$$

In the following section, the result given by equation (13) will be expressed in polar coordinates and used to solve for the displacement field produced by a CLoVER sector.

\section{GW excitation by a CLoVER sector}

The CLoVER sector geometry is easily described using polar coordinates, and is defined by the transducer's inner and outer radii, $R_{\mathrm{I}}$ and $R_{\mathrm{O}}$, as well as its left- and right-most angular edges, $\theta_{\mathrm{L}}$ and $\theta_{\mathrm{R}}$. Figure $4(\mathrm{a})$ shows a schematic of the transducer's geometry. At this point, equation (13) must be modified to express the displacement components in polar coordinates. This modification involves the use of the 2D Fourier transform for polar coordinates; for a generic function, $g(r, \theta)$, it is defined as:

$$
G(\xi, \phi)=\int_{0}^{\infty} \int_{0}^{2 \pi} g(r, \theta) \mathrm{e}^{\mathrm{i} \xi r \cos (\theta-\phi)} r \mathrm{~d} \theta \mathrm{d} r
$$

while the inverse transform is defined through:

$$
g(r, \theta)=\frac{1}{4 \pi^{2}} \int_{0}^{\infty} \int_{0}^{2 \pi} G(\xi, \phi) \mathrm{e}^{-\mathrm{i} \xi r \cos (\theta-\phi)} \xi \mathrm{d} \phi \mathrm{d} \xi .
$$

Using these definitions, along with the transformations $\xi_{x}=\xi \cos \phi$ and $\xi_{y}=\xi \sin \phi$, yields the following equation for the Cartesian displacement components expressed in polar coordinates:

$$
\begin{gathered}
\mathbf{u}_{A}(r, \theta, t)=\int_{0}^{2 \pi} \int_{0}^{\infty} \frac{\Gamma_{A}(\xi)}{D_{\mathrm{A}}(\xi)} \boldsymbol{\Psi}_{A}(\xi, \phi) \overline{\mathbf{F}}(\xi, \phi) \\
\times \mathrm{e}^{-\mathrm{i} \xi r \cos (\theta-\phi)} \mathrm{e}^{\mathrm{i} \omega t} \mathrm{~d} \xi \mathrm{d} \phi .
\end{gathered}
$$

The matrix $\Gamma_{A}$ is a $3 \times 3$ diagonal matrix of coefficients whose components are given by:

$$
\Gamma_{11}=\Gamma_{22}=\frac{\tau_{0} \sin \beta b \xi}{4 \pi^{2} \mu \beta \cos \beta b}
$$

$\Gamma_{33}=\frac{-\mathrm{i} \tau_{0} \xi^{2}}{4 \pi^{2} \mu}\left[2 \alpha \beta \cos \alpha b \sin \beta b+\left(\xi^{2}-\beta^{2}\right) \cos \beta b \sin \alpha b\right]$.

Similarly, $\Psi_{A}$ is a $3 \times 2$ matrix of coefficients defined through:

$$
\Psi_{A}=\left[\begin{array}{c}
-\gamma_{3}^{(1)}-\gamma_{4}^{(1)}\left(\mathrm{e}^{-2 \mathrm{i} \phi}+\mathrm{e}^{2 \mathrm{i} \phi}\right) \\
\gamma_{1}^{(2)}\left(\mathrm{e}^{2 \mathrm{i} \phi}-\mathrm{e}^{-2 \mathrm{i} \phi}\right) \\
\cos \phi \\
\gamma_{5}^{(1)}\left(\mathrm{e}^{2 \mathrm{i} \phi}-\mathrm{e}^{-2 \mathrm{i} \phi}\right) \\
-\gamma_{4}^{(2)}-\gamma_{5}^{(2)}\left(\mathrm{e}^{-2 \mathrm{i} \phi}+\mathrm{e}^{2 \mathrm{i} \phi}\right) \\
\sin \phi
\end{array}\right]
$$

where the coefficients $\gamma_{i}^{(j)}$ (defined in the appendix) depend on the substrate material properties, frequency, and wavenumbers. Finally, the vector $\overline{\mathbf{F}}$ contains the Fourier transform of the shear tractions produced at the transducer's edges. Only shear 
stresses along the piezoceramic fiber direction are considered, as shown in figure 4(b). This choice is based on the fact that the transducer is acting on the $3-3$ piezoelectric effect, which makes the strains induced along the fiber's axis significantly larger than those along its normal direction. For a typical piezoelectric material (such as PZT-5A) poled along the thickness direction, the piezoelectric coupling coefficient normal to the poling direction is approximately 54\% smaller than that along the poling direction [39]. This level of actuation is still significant, but the strains induced along this direction are further attenuated due to the high aspect ratio of the fiber and the construction of the APT device. As previously discussed, the APT is a composite transducer with epoxy located in between any two fibers. Thus, the shear lag effect effectively eliminates any strain transmitted normal to the fiber length. As a result, for the case of a CLoVER sector, the boundary conditions given by equation (3), transformed to polar coordinates, take the form:

$$
\begin{gathered}
\sigma_{z r}(r, \theta, b)=\tau_{0} f(r, \theta) \\
\sigma_{z z}(r, \theta, b)=\sigma_{z \theta}(r, \theta, b)=0
\end{gathered}
$$

where $\tau_{0}$ represents the amplitude of the traction exerted by the transducer on the substrate, and $f(r, \theta)$ is a function whose purpose is to make the stress non-zero only along the transducer's radial edges, as shown in figure 4(b). Such an expression is given by ${ }^{2}$ :

$$
f(r, \theta)=\left[u\left(\theta-\theta_{\mathrm{L}}\right)-u\left(\theta-\theta_{\mathrm{R}}\right)\right]\left[\delta\left(r-R_{\mathrm{I}}\right)-\delta\left(r-R_{\mathrm{O}}\right)\right] .
$$

In order to use the formulation presented above, the Fourier transform of equation (23) must be determined. Before doing so, the function must be decomposed along the $x$ - and $y$-directions to be compatible with the displacement vector given by equation (18). This is simply done by defining the $x$ and $y$-components as the function multiplied by an appropriate rotation matrix. Then, the necessary Fourier transforms are given by:

$$
\begin{aligned}
& \bar{F}_{1}(\xi, \phi)=\int_{0}^{2 \pi} \int_{0}^{\infty}\left[u\left(\theta-\theta_{\mathrm{L}}\right)-u\left(\theta-\theta_{\mathrm{R}}\right)\right] \\
& \quad \times\left[\delta\left(r-R_{\mathrm{I}}\right)-\delta\left(r-R_{\mathrm{O}}\right)\right] \cos \theta \mathrm{e}^{\mathrm{i} \xi r \cos (\theta-\phi)} r \mathrm{~d} r \mathrm{~d} \theta \\
& \bar{F}_{2}(\xi, \phi)=\int_{0}^{2 \pi} \int_{0}^{\infty}-\left[u\left(\theta-\theta_{\mathrm{L}}\right)-u\left(\theta-\theta_{\mathrm{R}}\right)\right] \\
& \quad \times\left[\delta\left(r-R_{\mathrm{I}}\right)-\delta\left(r-R_{\mathrm{O}}\right)\right] \sin \theta \mathrm{e}^{\mathrm{i} \xi r \cos (\theta-\phi)} r \mathrm{~d} r \mathrm{~d} \theta .
\end{aligned}
$$

The integrals given by equations (24) and (25) cannot be solved analytically. An alternate solution method is applicable since the radial and angular parts of the function are readily separable. For this type of functions, the Hankel transform of the radial part can be combined with a Fourier series representation of the angular part to obtain the desired Fourier transform [40]. In this way, the two-dimensional Fourier transform, $W(\xi, \phi)$, for a generic function $w(r, \theta)=g(\theta) h(r)$ can be expressed as:

$$
W(\xi, \phi)=\sum_{k=-\infty}^{\infty} c_{k} \mathrm{e}^{\mathrm{i} k \phi}(-\mathrm{i})^{k} \tilde{H}_{k}(\xi)
$$

2 Note that this definition of $f(r, \theta)$ yields correct units for stress as the delta function has units of [1/length] and the unit step function is dimensionless. where $\tilde{H}_{k}$ represents the $k$ th order Hankel transform of $h(r)$, defined through:

$$
\tilde{H}_{k}(\xi)=2 \pi \int_{0}^{\infty} h(r) J_{k}(r \xi) r \mathrm{~d} r
$$

and $c_{k}$ are the complex Fourier coefficients of the function $g(\theta)$, defined by:

$$
c_{k}=\frac{1}{2 \pi} \int_{0}^{2 \pi} g(\theta) \mathrm{e}^{-\mathrm{i} k \theta} \mathrm{d} \theta .
$$

In the case under consideration, the function $h(r)$ is defined by:

$$
h(r)=\delta\left(r-R_{\mathrm{I}}\right)-\delta\left(r-R_{\mathrm{O}}\right)
$$

while the two functions $g_{1}(\theta)$ and $g_{2}(\theta)$ are given by:

$$
\begin{array}{r}
g_{1}(\theta)=\left[u\left(\theta-\theta_{\mathrm{L}}\right)-u\left(\theta-\theta_{\mathrm{R}}\right)\right] \cos \theta \\
g_{2}(\theta)=-\left[u\left(\theta-\theta_{\mathrm{L}}\right)-u\left(\theta-\theta_{\mathrm{R}}\right)\right] \sin \theta .
\end{array}
$$

Therefore, the necessary Fourier transforms for the shear tractions applied on the substrate's surface result in:

$$
\bar{F}_{j 1}=\sum_{k=-\infty}^{\infty} c_{k}^{(j)} \mathrm{e}^{\mathrm{i} k \phi}(-i)^{k} \chi_{k}, \quad j=1,2
$$

where:

$$
\chi_{k}=2 \pi\left[R_{\mathrm{O}} J_{k}\left(\xi R_{\mathrm{O}}\right)-R_{\mathrm{I}} J_{k}\left(\xi R_{\mathrm{I}}\right)\right] .
$$

The resulting complex Fourier coefficients, obtained by substituting equations (30) into equation (28), can be expressed through:

$$
\begin{aligned}
c_{k}^{(1)} & =\frac{1}{2 \pi\left(1-k^{2}\right)}\left\{u\left(\theta_{\mathrm{L}}\right)\left[\mathrm{e}^{-\mathrm{i} k \theta_{\mathrm{L}}}\left(\mathrm{i} k \cos \theta_{\mathrm{L}}-\sin \theta_{\mathrm{L}}\right)-\mathrm{i} k\right]\right. \\
& \left.-u\left(\theta_{\mathrm{R}}\right)\left[\mathrm{e}^{-\mathrm{i} k \theta_{\mathrm{R}}}\left(\mathrm{i} k \cos \theta_{\mathrm{R}}-\sin \theta_{\mathrm{R}}\right)-\mathrm{i} k\right]\right\}, \\
& |k| \neq 1 \\
c_{k}^{(1)} & =\left[\left(2 \pi-\theta_{\mathrm{L}}-\sin \theta_{\mathrm{L}} \mathrm{e}^{-\mathrm{i} \theta_{\mathrm{L}}}\right) u\left(\theta_{\mathrm{L}}\right)\right. \\
& \left.-\left(2 \pi-\theta_{\mathrm{R}}-\sin \theta_{\mathrm{R}} \mathrm{e}^{-\mathrm{i} \theta_{\mathrm{R}}}\right) u\left(\theta_{\mathrm{R}}\right)\right][4 \pi]^{-1}, \\
& |k|=1 \\
c_{k}^{(2)}= & \frac{1}{2 \pi\left(1-k^{2}\right)}\left\{\left(\theta_{\mathrm{L}}\right)\left[\mathrm{e}^{-\mathrm{i} k \theta_{\mathrm{L}}}\left(\cos \theta_{\mathrm{L}}+\mathrm{i} k \sin \theta_{\mathrm{L}}\right)-1\right]\right. \\
& \left.-u\left(\theta_{\mathrm{R}}\right)\left[\mathrm{e}^{-\mathrm{i} k \theta_{\mathrm{R}}}\left(\cos \theta_{\mathrm{R}}+\mathrm{i} k \sin \theta_{\mathrm{R}}\right)-1\right]\right\}, \\
& |k| \neq 1 \\
c_{k}^{(2)} & =\left[u\left(\theta_{\mathrm{L}}\right)\left(-1+\mathrm{e}^{-2 \mathrm{i} \theta_{\mathrm{L}}}-4 \mathrm{i} \pi+2 \mathrm{i} \theta_{\mathrm{L}}\right)\right. \\
& \left.-u\left(\theta_{\mathrm{R}}\right)\left(-1+\mathrm{e}^{-2 \mathrm{i} \theta_{\mathrm{R}}}-4 \mathrm{i} \pi+2 \mathrm{i} \theta_{\mathrm{R}}\right)\right][8 \pi]^{-1}, \\
& |k|=1 .
\end{aligned}
$$

Since the CLoVER transducer is primarily meant to interrogate the structure away from the location where it is bonded, the main interest is in characterizing the GW field induced for radial positions such that $r>R_{\mathrm{O}}$, as shown in figure 5. This set is characterized by the fact that both edges of the transducer, inner and outer, send waves propagating in the positive radial direction (henceforth referred to as outward direction). The form of the solution, i.e., the combination of 


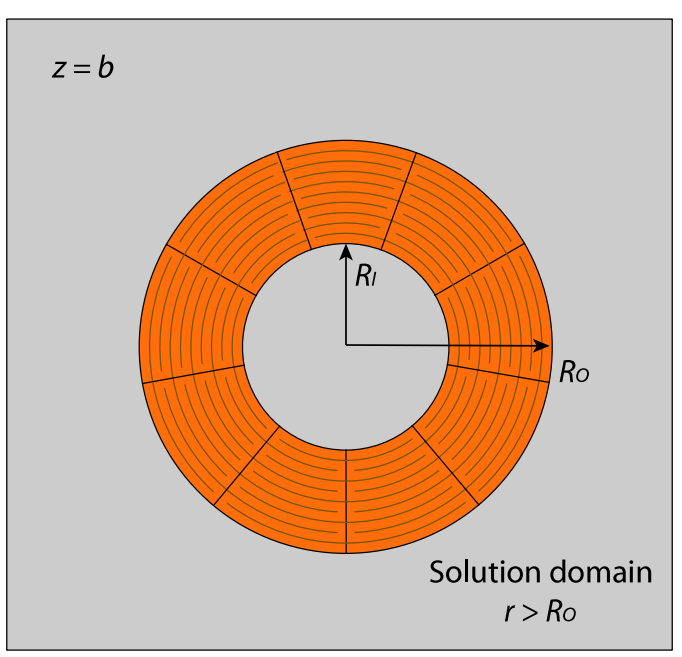

Figure 5. Schematic illustrating the solution domain.

equations (18) and (31), suggests that the integral definition of the Bessel function of $k$ th order be used:

$$
\begin{gathered}
J_{k}(\hat{z})=\frac{1}{2 \pi} \int_{0}^{2 \pi} \mathrm{e}^{-\mathrm{i} \frac{\pi}{2} k} \mathrm{e}^{\mathrm{i} \hat{z} \cos \phi} \mathrm{e}^{\mathrm{i} k \phi} \mathrm{d} \phi \\
=\frac{1}{2 \pi} \int_{0}^{2 \pi} \mathrm{e}^{\mathrm{i} \frac{\pi}{2} k} \mathrm{e}^{-\mathrm{i} \hat{z} \cos \phi} \mathrm{e}^{-\mathrm{i} k \phi} \mathrm{d} \phi .
\end{gathered}
$$

Note that equation (37) holds because the Bessel function returns a real number for $\hat{z}>0$. Therefore, taking the complex conjugate of the integrand does not alter the final result as its imaginary part is zero. This equation must be manipulated so that the exponential part of its integrand is similar to that in equation (18) (taking $\hat{z}=\xi r$ ). This process is carried out through the following change of variables:

$$
\phi=\bar{\phi}-\theta \text {. }
$$

This operation results in:

$$
J_{k}(\hat{z})=\frac{1}{2 \pi} \int_{\theta}^{2 \pi+\theta} \zeta_{k} \mathrm{e}^{-\mathrm{i} \hat{z} \cos (\theta-\bar{\phi})} \mathrm{e}^{-\mathrm{i} k \bar{\phi}} \mathrm{d} \bar{\phi}
$$

where:

$$
\zeta_{k} \equiv \mathrm{e}^{\mathrm{i} \frac{\pi}{2} k} \mathrm{e}^{\mathrm{i} k \theta} .
$$

The right-hand side in equation (39) is almost in the desired form. The only differences between this result and the form of equation (18) are in the integration limits and the index $k$ in the complex exponential. The difference in the integration limits is of no concern since the integrand of both functions is periodic in $\phi$ with a period of $2 \pi$. The indices in equation (18) will vary according to the powers of the exponentials given in equation (20). This can be simply resolved by redefining the resulting index of the complex exponential as $-k$, and incorporating this change in the corresponding multiplying coefficients. As a result, the solution to equation (18) in the angular wavenumber domain is given by:

$$
\begin{aligned}
& \mathbf{u}_{A}(r, \theta, t)=\int_{0}^{\infty} \sum_{k=-\infty}^{\infty}\left[\frac{2 \pi \Gamma_{A}(\xi)}{\zeta_{k}(\theta) D_{\mathrm{A}}(\xi)}\right. \\
& \left.\quad \times \Delta_{A}\left(k, \xi, R_{\mathrm{O}}, R_{\mathrm{I}}, \theta_{\mathrm{L}}, \theta_{\mathrm{R}}\right) J_{k}(\xi r)\right] \mathrm{e}^{\mathrm{i} \omega t} \mathrm{~d} \xi
\end{aligned}
$$

where $\Delta_{A}$ is a $3 \times 1$ column vector of coefficients that represents the source terms and is defined in the appendix. Note that the effect of the transducer dimensions are included in this term. The Bessel function solution presented in equation (41) corresponds to a standing wave. In order to obtain a propagating wave, we resort to the following definition of the Hankel function of the first and second kind:

$$
\begin{aligned}
& H_{k}^{(1)}(\hat{z})=J_{k}(\hat{z})+\mathrm{i} Y_{k}(\hat{z}) \\
& H_{k}^{(2)}(\hat{z})=J_{k}(\hat{z})-\mathrm{i} Y_{k}(\hat{z}) .
\end{aligned}
$$

Based on the frequency convention we have adopted, the Hankel function of the second kind corresponds to an outwardpropagating wave in time. Therefore only this part is retained, which yields:

$$
\mathbf{u}_{A}(r, \theta, t)=\int_{-\infty}^{\infty} \sum_{k=-\infty}^{\infty}\left[\frac{\pi \Gamma_{A}(\xi)}{\zeta_{k}(\theta) D_{\mathrm{A}}(\xi)} \Delta_{A} H_{k}^{(2)}(\xi r)\right] \mathrm{e}^{\mathrm{i} \omega t} \mathrm{~d} \xi .
$$

Note that the integration limits in the radial wavenumber domain have changed. This is because retaining the Hankel function of the second kind only is equivalent to replacing the azimuthal wavenumber integration limits from a range of $2 \pi$ to a range of $\pi$; hence, in order to keep the integration domain unchanged, the limits in the radial wavenumber domain must be modified ${ }^{3}$. The resulting integral is solved using the residue theorem from complex calculus. Since equation (43) is the quotient of two functions of $\xi$, it follows from the theory of complex calculus that the residue, $\bar{b}$, of this function at a pole $\xi_{\mathrm{A}}$ can be expressed as [41]:

$$
\bar{b}=\frac{N_{A}\left(\xi_{\mathrm{A}}\right)}{D_{\mathrm{A}}^{\prime}\left(\xi_{\mathrm{A}}\right)}
$$

where the' symbol indicates differentiation with respect to $\xi$, and the pole $\xi_{\mathrm{A}}$ corresponds to values of $\xi$ for which $D_{\mathrm{A}}$ vanishes; these points are the wavenumbers corresponding to the antisymmetric modes of Rayleigh-Lamb waves at a frequency $\omega$. The notation $N(\xi)$ has been used in equation (44) to illustrate the concept. This result can be combined with the residue theorem to express the solution of the integral in the $\xi$ domain as:

$$
\int_{-\infty}^{\infty} \frac{N(\xi)}{D(\xi)} \mathrm{d} \xi=2 \pi \mathrm{i} \sum_{\xi_{\mathrm{A}}} \frac{N\left(\xi_{\mathrm{A}}\right)}{D^{\prime}\left(\xi_{\mathrm{A}}\right)}, \quad \xi_{\mathrm{A}}>0
$$

where the condition that $\xi_{\mathrm{A}}$ be greater than zero indicates that only positive wavenumbers are to be included in the integration contour. Therefore, the solution may be expressed in final form as:

$$
\mathbf{u}_{A}(r, \theta, t)=\sum_{k=-\infty}^{\infty}\left[\frac{2 \pi^{2} \mathrm{i} \Gamma_{A}\left(\xi_{\mathrm{A}}\right)}{\zeta_{k}(\theta) D_{\mathrm{A}}^{\prime}\left(\xi_{\mathrm{A}}\right)} \Delta_{A} H_{k}^{(2)}\left(\xi_{\mathrm{A}} r\right)\right] \mathrm{e}^{\mathrm{i} \omega t} .
$$

Note that since only harmonic excitation is being considered, only one wavenumber needs to be included.

\footnotetext{
3 This statement is not fully rigorous as a correction term included in the integral definition of $H_{k}^{(2)}$ has been neglected. However, it can be shown that the contribution from this term is only significant for very small arguments.
} 
The solution given by equation (46) corresponds to the antisymmetric mode. The symmetric mode is given by a similar equation, which is derived by interchanging all sine and cosine terms whose arguments depend on the substrate half-thickness, $b$. This is a crucial change since it modifies the dispersion equation $D_{\mathrm{A}}$, which produces solutions with different wavenumbers.

In typical SHM applications, the structure to be inspected for damage is excited with a stress wave whose shape is determined by a time-dependent modulated signal. This type of signal is generally desired so as to control the frequency bandwidth and avoid dispersion. Hann-modulated signals have been successfully used in the past by several researchers and will be adopted in the present formulation [42]. Such a signal is given by:

$$
s(t)=\frac{1}{2}\left[1-\cos \left(2 \frac{\omega_{0} t}{n}\right)\right] \sin \left(\omega_{0} t\right)
$$

where $n$ is the number of half-cycles and $\omega_{0}$ represents the center frequency of excitation. In order to account for the timedependence, the time Fourier transform of equation (47) must be determined through:

$$
S(\omega)=\int_{-\infty}^{\infty} s(t) \mathrm{e}^{-\mathrm{i} \omega t} \mathrm{~d} t
$$

The time-dependent displacements are obtained through the inverse Fourier transform of the product of the transforms of the spatial and temporal parts. Since the excitation signal has several frequency components, a sum over all possible wavenumbers is necessary. Hence, the solution becomes (only antisymmetric mode is presented for simplicity):

$$
\begin{aligned}
& \mathbf{u}_{A}(r, \theta, t)=\frac{1}{2 \pi} \int_{-\infty}^{\infty} \sum_{\xi_{\mathrm{A}}}\left[\sum_{k=-\infty}^{\infty} \frac{\mathrm{i} \pi^{2} \Gamma_{A}\left(\xi_{\mathrm{A}}\right)}{\zeta_{k}(\theta) D_{\mathrm{A}}^{\prime}\left(\xi_{\mathrm{A}}\right)}\right. \\
& \left.\quad \times \Delta_{A} H_{k}^{(2)}\left(\xi_{\mathrm{A}} r\right)\right] S(\omega) \mathrm{e}^{\mathrm{i} \omega t} \mathrm{~d} \omega .
\end{aligned}
$$

\subsection{Additional spatial regions}

While the GW field induced by a CLoVER sector will be used for interrogation away from the transducer, it is still of interest to find a solution for the GW excitation problem in the remaining spatial regions. Therefore, region I is defined as the set of all points such that $r<R_{\mathrm{I}}$, as depicted in figure 6 . The solution for this region is obtained through an analogy to a circular transducer. In that case, the resulting wave pattern for radial positions within the edge of the actuator corresponds to standing waves. This result can be intuitively understood due to the symmetry of the source. Therefore, each source term in the Fourier expansion used to obtain the solution for a CLoVER sector will be treated as a standing wave. Once all the terms have been multiplied by the corresponding Fourier coefficient and summed together, the result will correspond to the GW field excited by a CLoVER sector. To emphasize, note that each term in the sum represents a standing wave, but the overall combination yields a propagating wave. Therefore, the expression for the antisymmetric displacement components

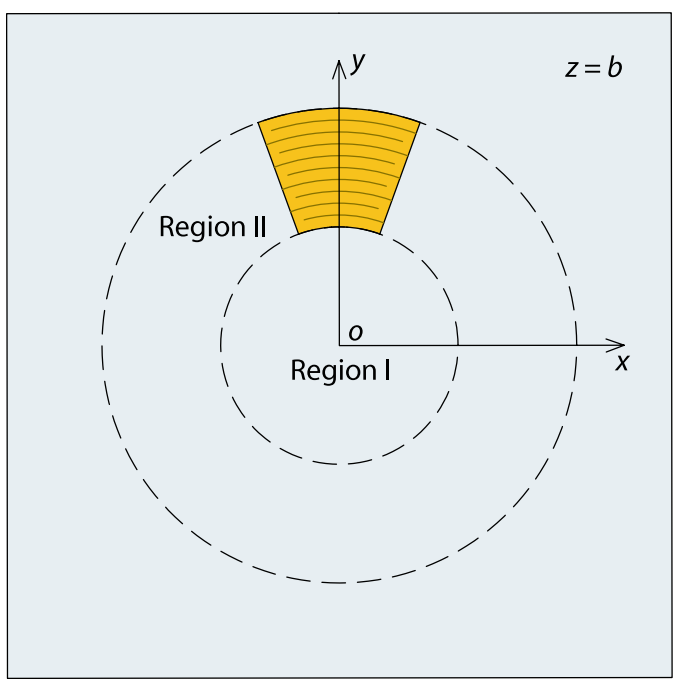

Figure 6. Additional solution regions for CLoVER GW excitation.

induced under harmonic excitation at a frequency $\omega$ is given by:

$$
\mathbf{u}_{A}=\sum_{k=-\infty}^{\infty} \frac{2 \mathrm{i} \pi^{2} \boldsymbol{\Gamma}_{A}\left(\xi_{\mathrm{A}}\right)}{\zeta_{k}(\theta) D_{\mathrm{A}}^{\prime}\left(\xi_{\mathrm{A}}\right)} \boldsymbol{\Lambda}_{A}\left(k, \xi_{\mathrm{A}}\right) J_{k}\left(\xi_{\mathrm{A}} r\right) \mathrm{e}^{\mathrm{i} \omega t}
$$

where $\Lambda_{A}$ is a $3 \times 1$ column vector of coefficients that represent the source terms, presented in the appendix.

In a manner analogous to region I, region II is defined as the set of all points such that $R_{\mathrm{I}}<r<R_{\mathrm{O}}$. This set of points is characterized by the fact that the each source contribution from the outer edge of the transducer corresponds to a standing wave, while those from the inner edge of the transducer correspond to traveling waves. Thus, the solution for this region is obtained as a combination of the two solutions presented previously. The antisymmetric displacement components under harmonic excitation at a frequency $\omega$ are given by:

$$
\begin{aligned}
\mathbf{u}_{A} & =\sum_{k=-\infty}^{\infty} \frac{2 \mathrm{i} \pi^{2} \boldsymbol{\Gamma}_{A}\left(\xi_{\mathrm{A}}\right)}{\zeta_{k}(\theta) D_{\mathrm{A}}^{\prime}\left(\xi_{\mathrm{A}}\right)}\left[\overline{\boldsymbol{\Upsilon}}_{A}\left(k, \xi_{\mathrm{A}}\right) J_{k}\left(\xi_{\mathrm{A}} r\right)\right. \\
& \left.-\Upsilon_{A}\left(k, \xi_{\mathrm{A}}\right) H_{k}^{(2)}\left(\xi_{\mathrm{A}} r\right)\right] \mathrm{e}^{\mathrm{i} \omega t}
\end{aligned}
$$

where $\Upsilon_{A}$ and $\bar{\Upsilon}_{A}$ are vectors of coefficients whose definition is given in the appendix. Note that $\Upsilon_{A}$ is associated with waves originating at the inner radial edge of the transducer, while $\bar{\Upsilon}_{A}$ corresponds to waves originating from its outer radial edge.

\section{Finite element verification}

Results from the theoretical formulation presented above were compared with three-dimensional FE simulations run in ABAQUS [43] to assess its spatial and temporal performance. Taking advantage of the problem's symmetries, only one quarter of an aluminum plate was considered. A summary of the material properties and actuator dimensions considered in the simulations is given in table 1 .

The mesh consisted of three-dimensional continuum elements, and was primarily composed of eight-node bricks 

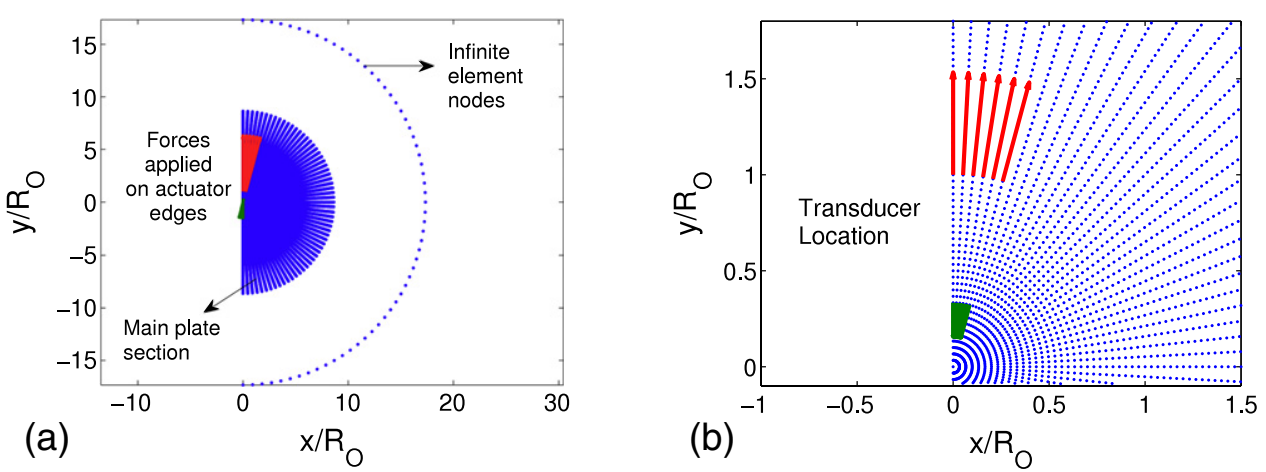

Figure 7. FE mesh: (a) complete overview; (b) detail on shear traction application points.

Table 1. Substrate material properties and actuator dimensions used in FE simulations and theoretical results.

\begin{tabular}{ll}
\hline Parameter & Value \\
\hline$E(\mathrm{GPa})$ & 70 \\
$v$ & 0.33 \\
$\rho\left(\mathrm{kg} \mathrm{m}^{-3}\right)$ & 2700 \\
$R_{\mathrm{O}}(\mathrm{m})$ & 0.015 \\
$R_{\mathrm{I}}(\mathrm{m})$ & 0.005 \\
$\Delta \theta(\mathrm{deg})$ & 30 \\
\hline
\end{tabular}

(C3D8). In addition, the geometry of the mesh required the use of six-node tetrahedra (C3D6) for the elements immediately connected to the origin. Similarly, infinite continuum elements (CIN3D8) were used on the outer radial boundary of the model, in an effort to minimize boundary reflections [43]. However, the results showed that these elements were not successful in achieving this, and therefore the radial positions selected for comparison were located far from the radial edge. In all cases, three elements were used through the thickness of the plate. A schematic of the mesh is shown in figure 7(a), while its relevant dimensions (radius, $R$, and half-thickness, $b$ ) are given in table 2. Symmetric $\left(\mathrm{S}_{0}\right)$ and antisymmetric $\left(\mathrm{A}_{0}\right)$ modes were excited by specifying a symmetry and antisymmetry condition with respect to the $z$-axis, respectively. The symmetric mode was used to validate the out-of-plane displacement, while the antisymmetric mode was used to model the in-plane displacements. This choice was based on the fact that the antisymmetric mode has a higher frequency threshold for the appearance of the SH-mode (shear horizontal mode present only in the in-plane displacements), which is not considered in the theoretical solution and would therefore prevent an accurate verification.

The radial dimension of the elements, $\Delta r_{\mathrm{e}}$, was selected so as to have at least 20 nodes per wavelength for the highest frequency of the toneburst excitation. The element azimuthal size, $\Delta \theta_{\mathrm{e}}$, was selected at $3^{\circ}$ providing six nodes along the angular span of the actuator, whose centerline was located at $90^{\circ}$. A summary of the mesh parameters is presented in table 2. The shear tractions caused by the actuator were modeled through nodal forces on the nodes corresponding to the actuator edges. Since the theoretical model considers the traction per unit length to be constant along the radial edges of the transducer, the relative force amplitudes must
Table 2. Mesh and analysis parameters used in FE simulations.

\begin{tabular}{lll}
\hline Parameter & Value $\left(\mathrm{S}_{0}\right)$ & Value $\left(\mathrm{A}_{0}\right)$ \\
\hline$R(\mathrm{~m})$ & 0.54 & 0.13 \\
$\Delta r_{\mathrm{e}}(\mathrm{m})$ & 0.0025 & 0.0005 \\
$\Delta \theta_{\mathrm{e}}(\mathrm{deg})$ & 3 & 3 \\
$\Delta t(\mathrm{~s})$ & $10^{-7}$ & $10^{-7}$ \\
$b(\mathrm{~m})$ & 0.002 & 0.002 \\
$f_{0}(\mathrm{kHz})$ & 100 & 100 \\
$n$ & 7 & 7 \\
No. of elements & 38700 & 47336 \\
No. of nodes & 52464 & 63444 \\
\hline
\end{tabular}

be scaled appropriately. This was achieved by considering point forces of unit magnitude on the outer radial edge and scaling the magnitude on the inner edge accordingly, which results in its amplitude being $R_{\mathrm{I}} / R_{\mathrm{O}}$. The time-dependent part of this forcing function was a Hann-modulated toneburst whose properties are also summarized in table 2. A schematic showing the details of the shear traction application is given in figure 7(b). The time step, $\Delta t$, was selected so as to satisfy the following criteria: (i) proper sampling of the highest frequency component of the excitation signal, and (ii) sufficient resolution of the time needed for the fastest traveling wave to move across one element in the radial direction [44]. An implicit dynamic analysis was performed with 1404 steps in all cases.

The numerical implementation of the theoretical solution requires that a finite number of terms in the infinite sum given by equation (49) be selected. This number was chosen based on the normalized error between the angular part of the shear traction function, equation (30), and its Fourier series representation, equation (26). This error is defined as:

$$
\varepsilon=\frac{\sqrt{\int_{0}^{2 \pi}\left|g_{1,2}-g_{1,2}^{\mathrm{F}}\right|^{2} \mathrm{~d} \theta}}{\sqrt{\int_{0}^{2 \pi} g_{1,2}^{2} \mathrm{~d} \theta}}
$$

where the superscript $F$ refers to the Fourier series representation of the function. Figure 8 shows how this error decreases with increasing number of terms. Based on this result, the sum was truncated at 150 terms as the reduction in error with increasing terms was slower after this point. In addition, the solution was implemented using a larger number of terms and no difference was observed, indicating 

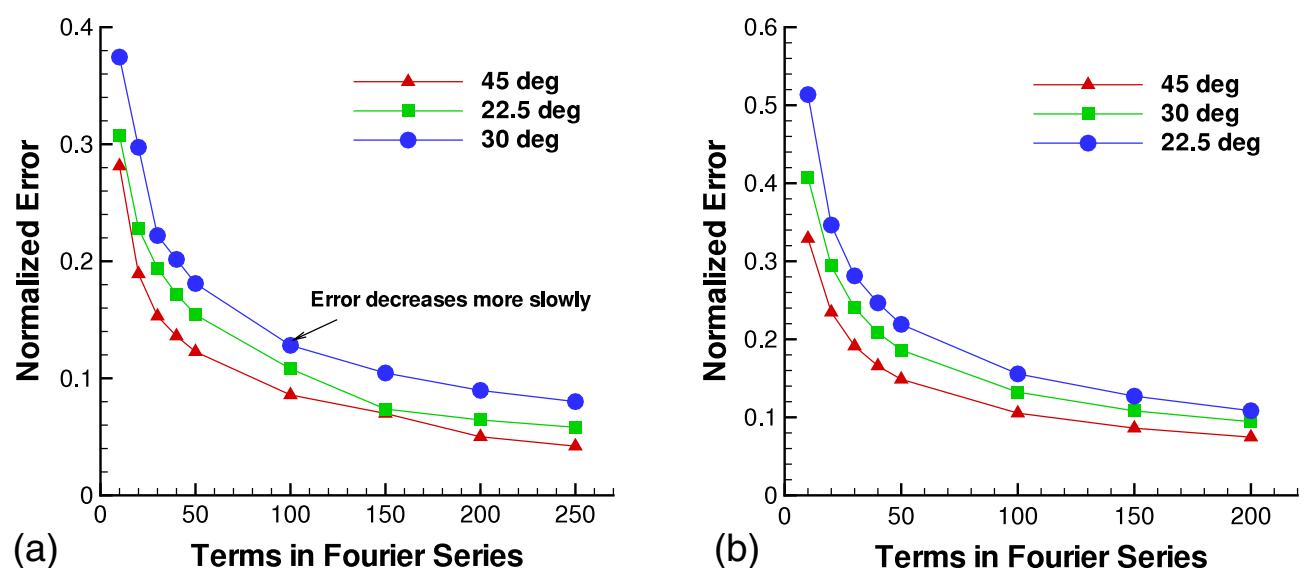

Figure 8. Normalized error between angular part of shear traction function and its Fourier series representation for: (a) $x_{1}$-component; (b) $x_{2}$-component.

this selection was sufficient for convergence. For the spatial comparisons, several azimuthal positions were selected at different radial locations sufficiently far from the radial edge to avoid boundary reflections. Figure 9(a) shows the set of points selected for for this. The radial positions selected were ten times the transducer's outer radius $(\hat{n}=10)$ for the symmetric mode, and five thirds times the outer radius $(\hat{n}=$ $5 / 3$ ) for the antisymmetric mode. Seven azimuthal locations from the transducer's centerline to its opposite direction were selected, which resulted in intervals of $30^{\circ}$. Figure 9 (b) shows the normalized peak-to-peak comparison between the theory and FE results, while figures 9(c) and (d) show a similar result for the in-plane displacements. Similarly, figures 10(a) and (b) show sample time histories for two different azimuthal positions. These figures indicate that the spatial distribution is accurately modeled by the theoretical solution.

A similar comparison was performed for regions I and II, where only the antisymmetric mode was considered due to its shorter wavelength. Figure 11(a) shows a schematic of the points selected for comparison. For region I, an azimuthal distribution similar to the one used in the previous case was employed, but the radial position was changed to one-half the transducer's inner radius. In region II, only six azimuthal points were considered to avoid having a point over the transducer's area, while the radial location selected was the transducer's midpoint along the radial direction. Figures 11(b) and (c) show good spatial correlation for the out-of-plane displacement in regions I and II, respectively. Similarly, figure 11(d) shows that the time history of the analytical displacements also matches very well with FE results.

\section{Sensor response}

In GW-based testing, a stress wave is excited by the piezoelectric actuator through the structural element whose condition is to be inspected. This wave typically experiences changes in its amplitude, frequency content, and group speed due to its interaction with any defects it encounters (e.g., cracks, corrosion, delaminations). Furthermore, these interactions produce additional waves that, generally, scatter in every direction. This process leads to the use of two primary methods of testing: the pitch-catch and pulse-echo methods. The pitch-catch method is based on identifying damage based on the changes that defects introduce into the wave by locating a sensor a certain distance away from the actuator and recording the received wave. In contrast, the pulse-echo method uses the reflections scattered from defects to identify and locate damage. This typically involves using a transducer that acts both as actuator and sensor. In either method, information about the damage is obtained from strains sensed by the piezoelectric transducer and the corresponding voltage signal generated through the piezoelectric effect. This voltage signal is then used to determine information about damage presence, location, and severity using adequate signal processing techniques. Therefore, it is necessary to relate the strains sensed by the transducer to the induced voltage. This will allow us to identify excitation frequencies and transducer dimensions that maximize the sensor response. Raghavan and Cesnik [13] proposed a model to do this by modeling the sensor as a capacitor. Using the assumption that the sensor is under plane stress conditions, the following expression was presented:

$$
V_{\mathrm{s}}=\frac{k \epsilon_{0} E_{s} \bar{t}_{s} g}{\left(1-v_{s}\right) C_{\mathrm{s}}} \int_{A_{s}} \epsilon_{i i} \mathrm{~d} A
$$

where the piezoelectric constant to be used depends on the relative directions of the applied electric field and the induced strain. Similarly, the capacitance of the sensor, $C_{\mathrm{s}}$, will depend on whether it is a uniform piezo material or an APT. An implication of this model is that the sensor is assumed to be infinitely compliant, so that it does not disturb the GW field produced by the actuator. The strains necessary to obtain the sensor response can be obtained from the displacement components defined previously by means of a linear straindisplacement relation. In polar coordinates, this is expressed as:

$$
\begin{gathered}
\epsilon_{r r}=\frac{\partial u_{\mathrm{r}}}{\partial r} \\
\epsilon_{\theta \theta}=\frac{u_{\mathrm{r}}}{r}+\frac{1}{r} \frac{\partial u_{\theta}}{\partial \theta}
\end{gathered}
$$




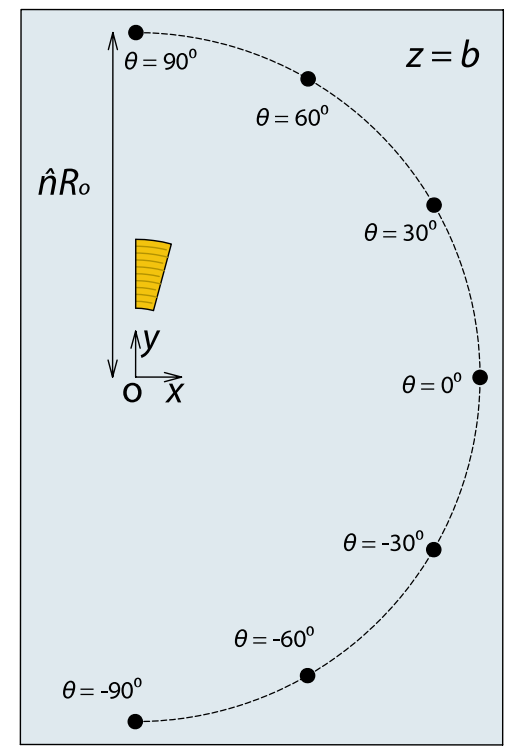

(a)

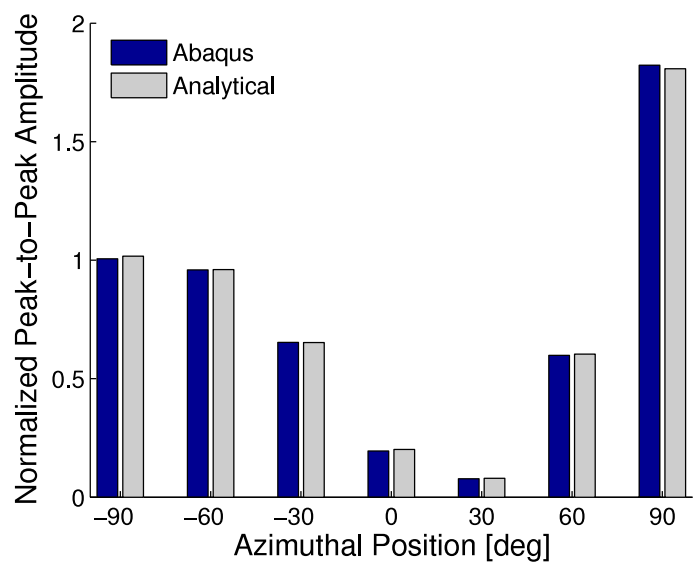

(c)

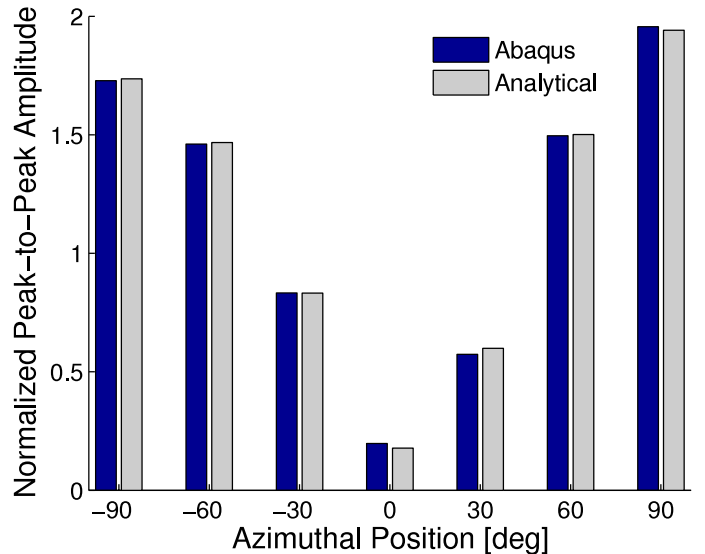

(b)

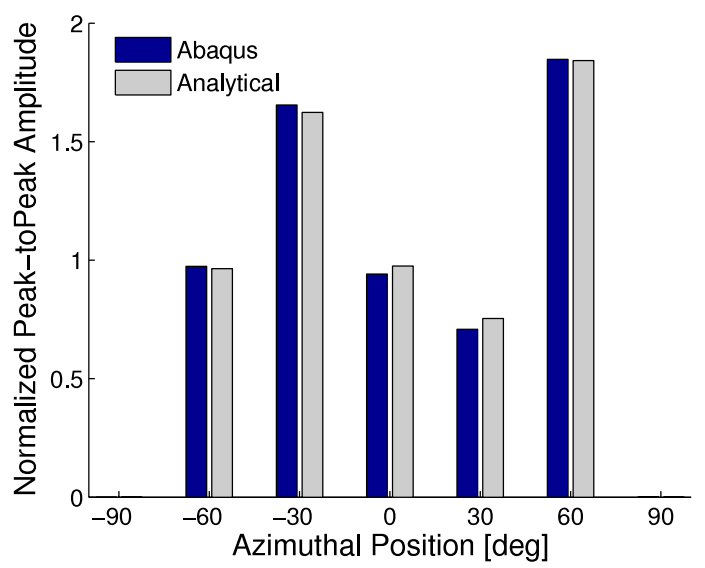

(d)

Figure 9. Comparison between FE and theoretical results. (a) Points selected for comparison. (b) Amplitude comparison for symmetric $u_{3}$. (c) Amplitude comparison for antisymmetric $u_{2}$. (d) Amplitude comparison for antisymmetric $u_{1}$.
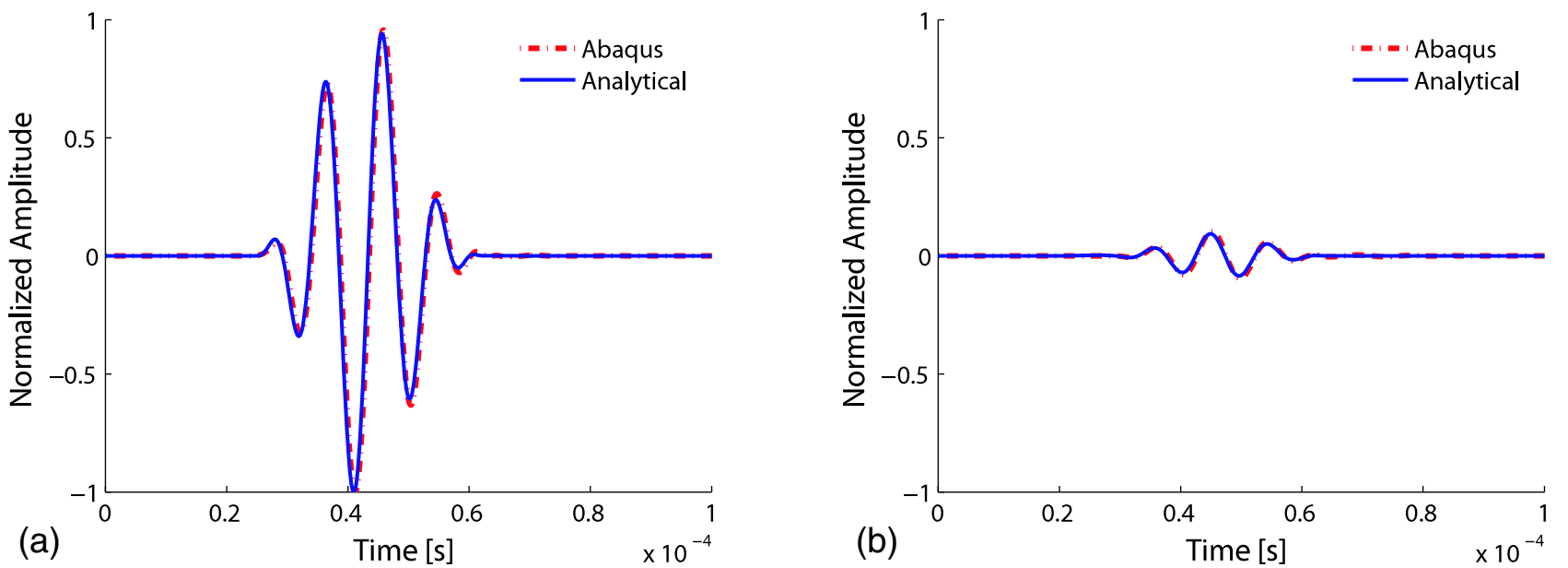

Figure 10. Sample time history for out-of-plane displacement at $r=10 R_{\mathrm{O}}$ (with baseline $R_{\mathrm{O}}=15 \mathrm{~mm}$ ) and $\theta=(\mathrm{a}) 90^{\circ}$ and (b) $0^{\circ}$. 


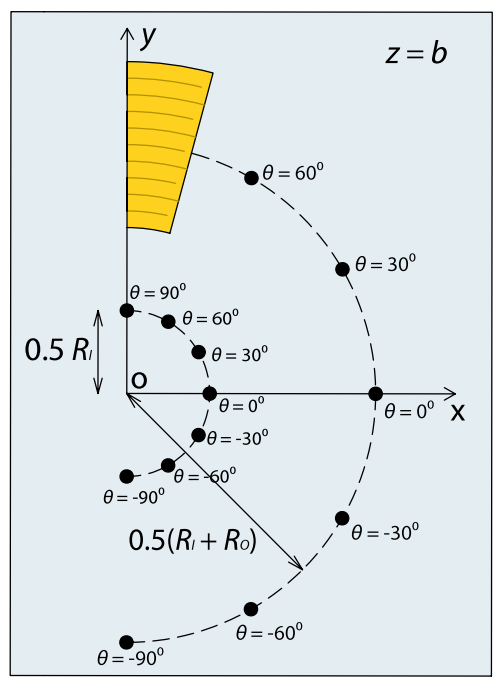

(a)

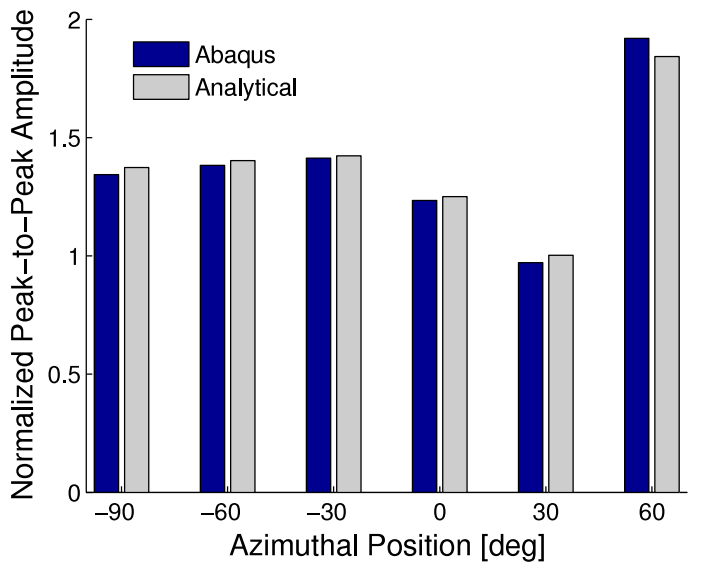

(c)

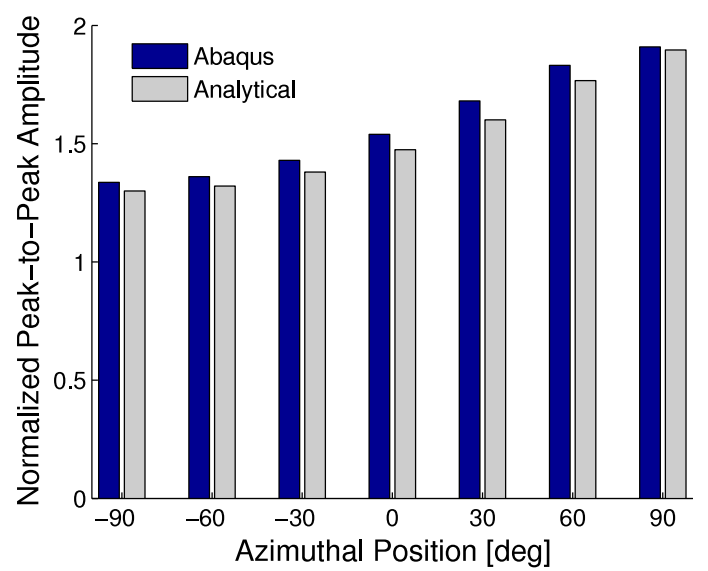

(b)

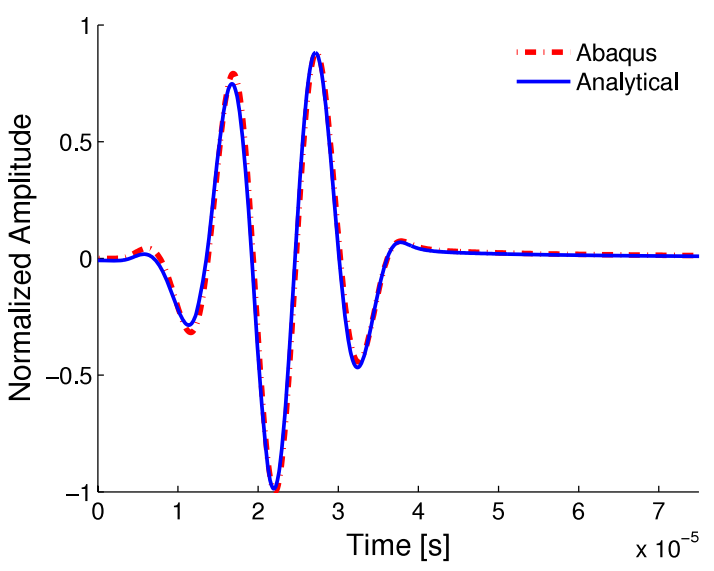

(d)

Figure 11. Comparison between FE and analytical results in regions I and II. (a) Points selected for comparison; (b) amplitude comparison for antisymmetric $u_{3}$ in region I; (c) amplitude comparison for antisymmetric $u_{3}$ in region II; (d) sample time history for out-of-plane displacement at the origin.

where $u_{\mathrm{r}}$ and $u_{\theta}$ are the radial and azimuthal displacement components, respectively. These components can be expressed in terms of the Cartesian components obtained previously through the transformation:

$$
\left\{\begin{array}{l}
u_{\mathrm{r}} \\
u_{\theta}
\end{array}\right\}=\left[\begin{array}{cc}
\cos \theta & \sin \theta \\
-\sin \theta & \cos \theta
\end{array}\right]\left\{\begin{array}{l}
u_{1} \\
u_{2}
\end{array}\right\} .
$$

Since the Cartesian components were previously obtained in terms of polar coordinates, it follows from equation (56) that:

$$
\frac{\partial u_{\mathrm{r}}}{\partial r}=\frac{\partial u_{1}}{\partial r} \cos \theta+\frac{\partial u_{2}}{\partial r} \sin \theta
$$

and

$$
\frac{\partial u_{\theta}}{\partial \theta}=\cos \theta\left(\frac{\partial u_{2}}{\partial \theta}-u_{1}\right)-\sin \theta\left(\frac{\partial u_{1}}{\partial \theta}+u 2\right) .
$$

Similarly, the strain-displacement relation in Cartesian coordinates is given by:

$$
\begin{gathered}
\epsilon_{x x}=\frac{\partial u_{1}}{\partial x} \\
\epsilon_{y y}=\frac{\partial u_{2}}{\partial y} .
\end{gathered}
$$

Since the displacement components were found in terms of polar coordinates, the coordinate transformation $r=$ $\sqrt{x^{2}+y^{2}}, \theta=\tan ^{-1}(y / x)$ is used along with the chain rule of derivatives to obtain:

$$
\begin{aligned}
\epsilon_{x x} & =\frac{\partial u_{1}}{\partial r} \cos \theta-\frac{\partial u_{1}}{\partial \theta} \frac{\sin \theta}{r} \\
\epsilon_{y y} & =\frac{\partial u_{2}}{\partial r} \sin \theta+\frac{\partial u_{2}}{\partial \theta} \frac{\cos \theta}{r} .
\end{aligned}
$$

Finally, the $r$ and $\theta$ derivatives of the displacement components, for the antisymmetric mode, may be expressed 


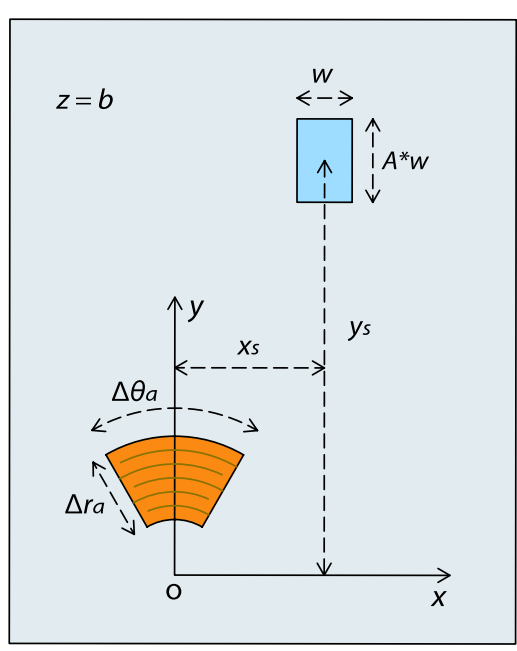

(a)

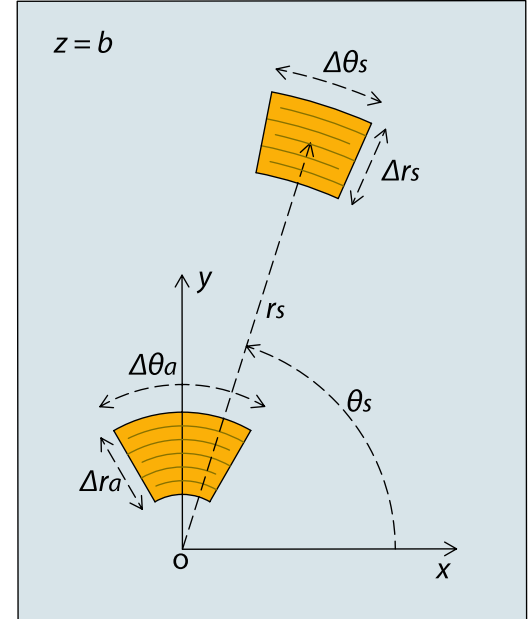

(b)

Figure 12. Sensor geometry and location used in analysis. (a) Rectangular piezo sensor. (b) Rectangular APT sensor.

in vector form as:

$$
\frac{\partial \mathbf{u}_{A}}{\partial r}=\sum_{k=-\infty}^{\infty}\left[\frac{\mathrm{i} \pi^{2} \xi_{\mathrm{A}} \boldsymbol{\Gamma}_{A}\left(\xi_{\mathrm{A}}\right)}{\zeta_{k}(\theta) D_{\mathrm{A}}^{\prime}\left(\xi_{\mathrm{A}}\right)} \Delta_{A}\left\{H_{k-1}^{(2)}\left(\xi_{\mathrm{A}} r\right)-H_{k+1}^{(2)}\left(\xi_{\mathrm{A}} r\right)\right\}\right]
$$

and:

$$
\frac{\partial \mathbf{u}_{A}}{\partial \theta}=\sum_{k=-\infty}^{\infty}\left[\frac{-2 k \pi^{2} \boldsymbol{\Gamma}_{A}\left(\xi_{\mathrm{A}}\right)}{\zeta_{k}(\theta) D_{\mathrm{A}}^{\prime}\left(\xi_{\mathrm{A}}\right)} \boldsymbol{\Delta}_{A} H_{k}^{(2)}\left(\xi_{\mathrm{A}} r\right)\right] .
$$

\subsection{Rectangular piezo sensor}

The sensor response of a homogeneous rectangular piezoelectric sensor is of practical interest in SHM applications. Consider a rectangular sensor of width $w$, and aspect ratio $A^{*}$, so that, $h=A^{*} w$ and $A_{s}=A^{*} w^{2}$, as shown in figure 12(a). The sensor is centered at the point $x=x_{s}, y=y_{s}$ and is under the GW field excited by a CLoVER sector of radial span $\Delta r$ and angular span $\Delta \theta$ centered at the point $r_{\mathrm{a}}=0.5\left(R_{\mathrm{I}}+R_{\mathrm{O}}\right)$, $\theta_{\mathrm{a}}=90^{\circ}$. In contrast to the case of an APT sensor, the piezo material has isotropic piezoelectric properties in the plane normal to the poling direction, and therefore senses all in-plane extensional strains. In addition, this enables the piezo to be modeled as a parallel plate capacitor filled with a dielectric material. In this way, its capacitance may be expressed through:

$$
C_{\mathrm{s}}=\frac{k \epsilon_{0} A_{s}}{\bar{t}_{s}} .
$$

Consequently, its sensor response is given by [13]:

$$
V_{\mathrm{s}}=\frac{E_{s} \bar{t}_{s} g_{13}}{A_{s}\left(1-v_{s}\right)} \int_{y_{s}-A^{*} \frac{w}{2}}^{y_{s}+A^{*} \frac{w}{2}} \int_{x_{s}-\frac{w}{2}}^{x_{s}+\frac{w}{2}}\left(\epsilon_{x x}+\epsilon_{y y}\right) \mathrm{d} x \mathrm{~d} y
$$

where $\epsilon_{x x}$ and $\epsilon_{y y}$ are defined by equations (61) and (62), respectively.

Note that equation (66) holds for harmonic forcing at a frequency $\omega$. For the general case of a time-dependent excitation, such as the toneburst signal considered before, the integrand is multiplied by the time Fourier transform of this signal and integrated over an infinite frequency range (which in reality is limited to the frequency bandwidth of the excitation toneburst), as outlined in section 4. However, in order to identify optimal excitation frequencies and sensor dimensions a harmonic analysis is sufficient. Once these have been identified, they can be used as center frequencies of timedependent excitations.

The variation in three parameters was examined. First, we explored the excitation frequencies to determine values at which the sensor response would be maximum, as well as to identify values that should be avoided due to negligible sensing response. Second, two sensor dimensions were explored. The width of the sensor was considered, which resulted in the definition of the non-dimensional parameter $w^{*}$ as $w / \Delta r$. The effect of the sensor's aspect ratio was also studied. The results from this analysis are shown in figures 13 and 14. In the study of the aspect ratio, the value of $w^{*}$ was kept fixed at 1 . The two modes show different qualitative behavior over the range of frequencies studied with the $\mathrm{A}_{0}$ mode showing two main frequencies at which high response is obtained, as opposed to the $\mathrm{S}_{0}$ case which shows primarily one up to $500 \mathrm{kHz}$. Note that both dimensions are critical in the response of the rectangular piezoelectric sensor, with smaller sizes resulting in better performance. This observation is consistent with the findings reported in [13]. In addition to the decrease in amplitude observed for larger sensors, it can also be seen that the number of nodes increases significantly.

\subsection{Rectangular APT sensor}

The response of a rectangular APT sensor under the GW field excited by a CLoVER sector is now derived. This analysis is based on the assumption that only extensional strains along the piezoelectric fiber direction are sensed. This is a reasonable simplification since the induced shear strains are small compared to the extensional ones. Furthermore, as discussed in section 4 , the piezoelectric performance of 

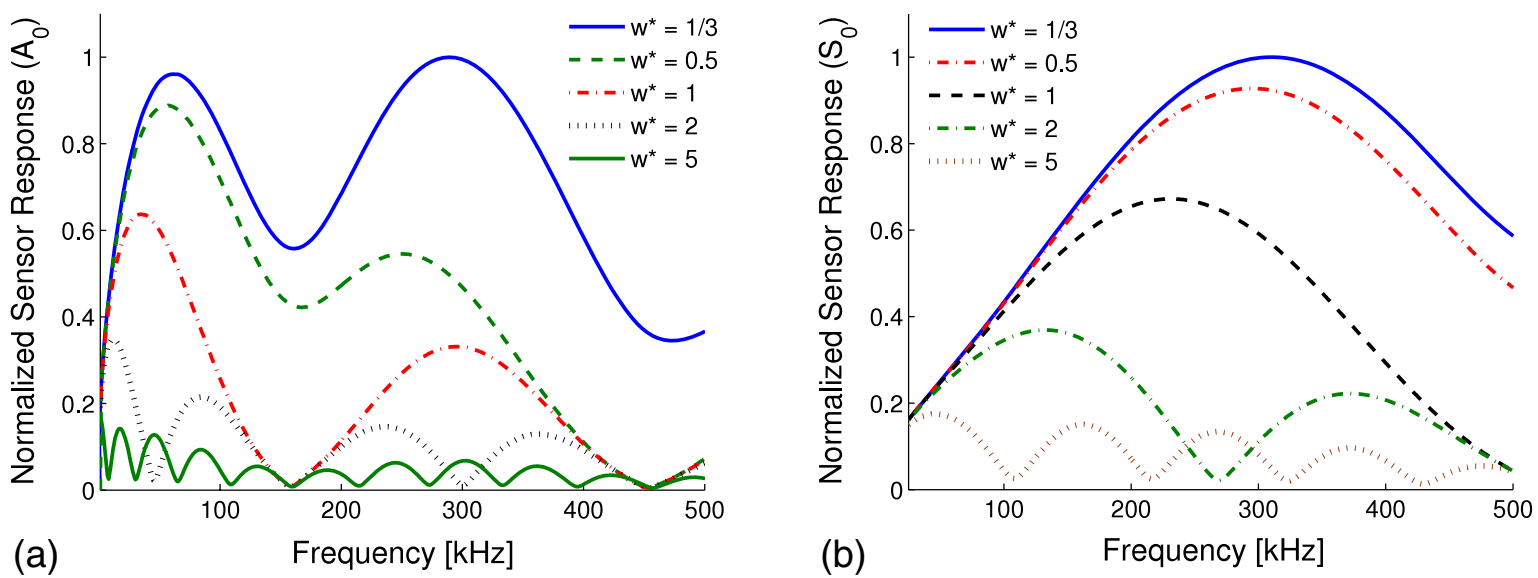

Figure 13. Effect of sensor width on piezo sensor response. The baseline case was $\Delta r=0.005$ m. (a) $\mathrm{A}_{0}$ mode. (b) $\mathrm{S}_{0}$ mode.
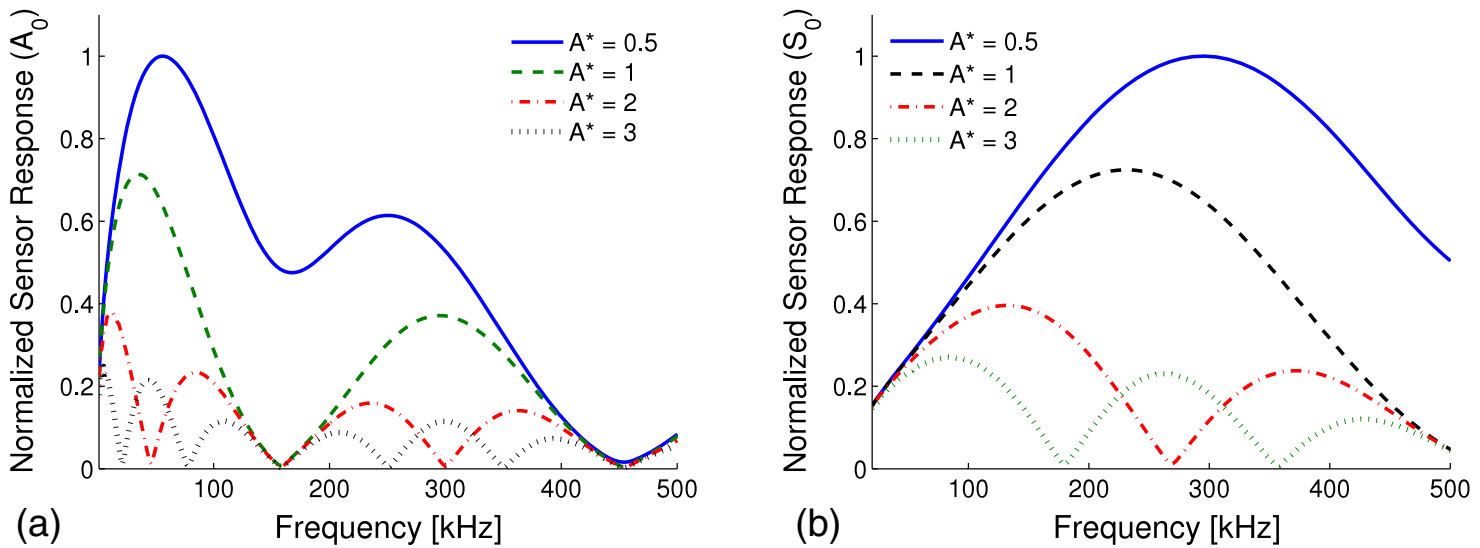

Figure 14. Effect of sensor aspect ratio $\left(A^{*}\right)$ on piezo sensor response. The baseline case was $\Delta r=0.005$ m. (a) $\mathrm{A}_{0}$ mode. (b) $\mathrm{S}_{0}$ mode.

the transducer along the normal direction to the fiber is very weak. Therefore, for the rectangular configuration, only $\epsilon_{r r}$ is needed. The rectangular sensor is modeled by using a wedgeshaped geometry whose radial origin coincides with that of the CLoVER actuator. Therefore, while its effective geometry is that of a rectangle, its dimensions will be expressed in terms of radial and azimuthal components. This further assumes that the fibers in the rectangular device are always oriented along the radial direction. Consider a rectangular APT sensor centered at the point $r=r_{s}, \theta=\theta_{s}$, with radial dimension $\Delta r_{s}$, angular span $\Delta \theta_{s}$, and surface area $A_{s}=r_{s} \Delta r_{s} \Delta \theta_{s}$, as shown in figure 12(b). The sensor is subjected to the GW field excited by a CLoVER sector with radial dimension $\Delta r$ and angular span $\Delta \theta$ centered at the point $r_{\mathrm{a}}=0.5\left(R_{\mathrm{I}}+R_{\mathrm{O}}\right), \theta_{\mathrm{a}}=90^{\circ}$. In order to use the sensor response equation presented earlier (equation (53)), an expression for the APT capacitance is needed. For this type of transducer, the capacitance is typically analyzed using the repetitive nature of the interdigitated electrode pattern. In this way, only the electric field in a representative unit cell, defined as the region between any two electrode fingers, is considered. A comprehensive study on this electric field was conducted by Lloyd [45] using conformal mapping techniques. In that work, it was shown that the capacitance of each unit cell was primarily determined by the piezoceramic thickness, electrode finger width, and electrode finger spacing, while it scaled linearly with electrode finger length. Thus, the capacitance of a unit cell can be expressed as:

$$
C_{\mathrm{uc}}=p\left(k, \epsilon_{0}, d_{\mathrm{IDE}}, \bar{t}_{a}\right) L_{\mathrm{IDE}}
$$

where, in the case of a rectangular APT, the electrode finger length is determined by its radial position, $r_{\mathrm{IDE}}$, and the azimuthal span, $\Delta \theta$, so that:

$$
C_{\mathrm{uc}}=p\left(k, \epsilon_{0}, d_{\mathrm{IDE}}, \bar{t}_{a}\right) r_{\mathrm{IDE}} \Delta \theta .
$$

A closed form expression for the function $p$ cannot be readily obtained. However, the work of Lloyd [45] showed that this function is non-linearly dependent on the electrode finger and unit cell geometry, and that it increases exponentially as the center-to-center distance between the electrode fingers approaches zero. It is also important to note that any contribution from the capacitance of the epoxy has been neglected. This is a logical choice since its dielectric constant $(k \sim 6)$ [46] is much smaller than that for a typical piezoelectric ceramic $(k \sim 1700$, PZT-5A) [39]. The capacitance of the overall device may be obtained by considering it as a composition of capacitors connected in 

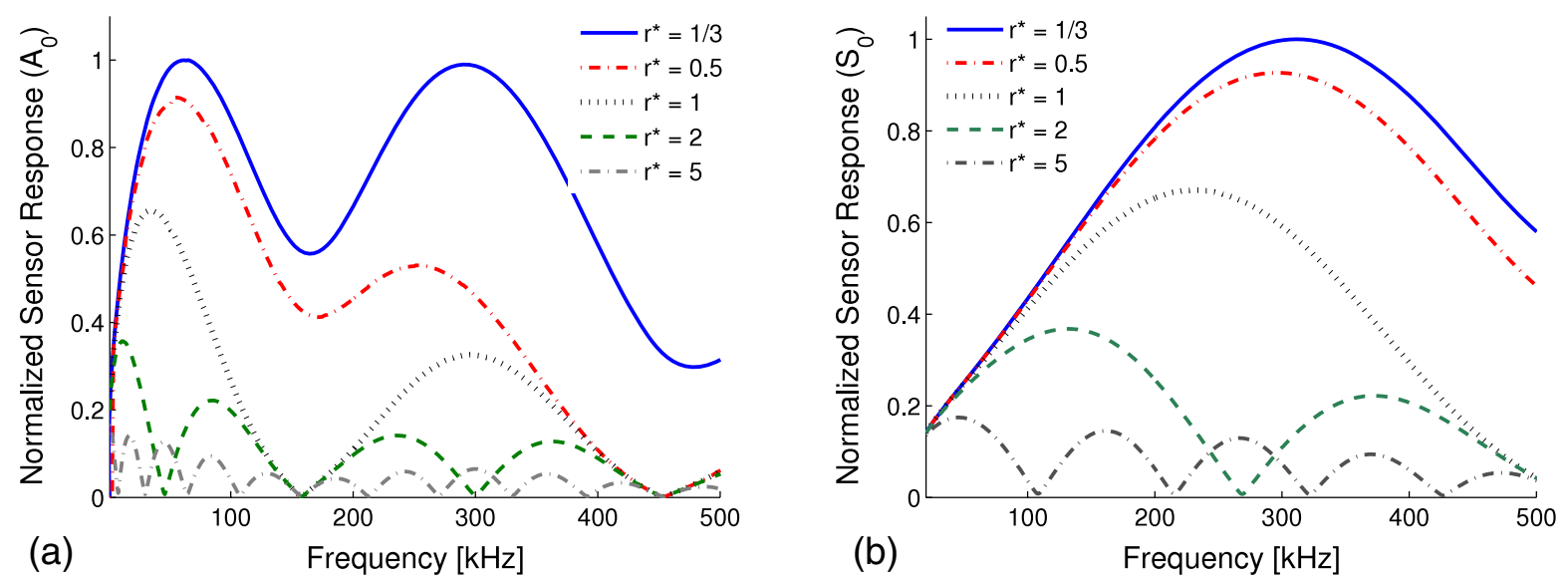

Figure 15. Effect of radial dimension on rectangular APT sensor response. The baseline case was $\Delta r=0.005$ m. (a) $\mathrm{A}_{0}$ mode. (b) $\mathrm{S}_{0}$ mode.

parallel. Then, the capacitance of each unit cell may be simply added together which results in:

$$
C=p\left(k, \epsilon_{0}, d_{\mathrm{IDE}}, \bar{t}_{a}\right) \Delta \theta \frac{R_{\mathrm{O}}+R_{\mathrm{I}}}{2} \Delta r
$$

Using equation (69), the voltage induced in the APT sensor due to the induced strains is given by:

$$
V_{\mathrm{s}}=\frac{k \epsilon_{0} E_{s} \bar{t}_{s} g_{33}}{\left(1-v_{s}\right) p A_{s}} \int_{\theta_{s}-\frac{\Delta \theta_{s}}{2}}^{\theta_{s}+\frac{\Delta \theta_{s}}{2}} \int_{r_{s}-\frac{\Delta r_{s}}{2}}^{r_{s}+\frac{\Delta r_{s}}{2}} \epsilon_{r r} r \mathrm{~d} r \mathrm{~d} \theta
$$

Note that in the case of an APT, $g_{33}$ is used. In addition, for a given APT device, the function $p$ is a constant. Using the definition in equation (70), the voltage is given by:

$$
V_{\mathrm{s}}=\frac{k \epsilon_{0} E_{s} \bar{t}_{s} g_{33}}{\left(1-v_{s}\right) p A_{s}} \int_{\theta_{s}-\frac{\Delta \theta_{s}}{2}}^{\theta_{s}+\frac{\Delta \theta_{s}}{2}} \int_{r_{s}-\frac{\Delta r_{s}}{2}}^{r_{s}+\frac{\Delta r_{s}}{2}}\left[\frac{\partial u_{1}}{\partial r} \cos \theta+\frac{\partial u_{2}}{\partial r} \sin \theta\right]
$$$$
\times r \mathrm{~d} r \mathrm{~d} \theta \text {. }
$$

This analysis was performed in two separate steps; the ratio of the radial dimension was first explored by defining the non-dimensional parameter $r^{*}$ as the ratio $\Delta r_{s} / \Delta r$ and obtaining the sensor response, given by equation (71), over a wide frequency range for both symmetric and antisymmetric modes. The result from this analysis is shown in figure 15 . The location of the sensor was set to $r_{s}=15 R_{\mathrm{O}}$ and $\theta_{s}=$ $90^{\circ}$; this azimuthal position was selected since it coincides with the transducer's centerline which is the intended scanning direction. The actuator dimensions were similar to the ones presented in table 1 . In these and subsequent sensor response plots, the results presented are normalized by the maximum value in each set. Similar qualitative differences between the two modes are observed in this case, with the antisymmetric mode showing the lower response at a higher frequency than in the previous case.

The effect of varying the ratio of azimuthal spans was also examined, which resulted in the definition of the parameter $\theta^{*}$ as $\Delta \theta_{s} / \Delta \theta$. The results from this analysis, for $r^{*}$ fixed at 1 , are shown in figure 16. As it can be seen, this characteristic does not affect the trend observed in frequency (figure 15), but instead causes a decrease in amplitude. Notice that this effect is significant only if the actuator is made much smaller than the sensor or vice versa. These trends are logical since it is expected that changing the sensor dimension in the wave propagation direction will have the most significant effect. As in the previous case, it is evident that smaller sensors produce better results.

\section{Experimental studies on GW excitation by a CLoVER sector}

\subsection{CLoVER fabrication}

The CLoVER transducers used in this study were manufactured based on an adaptation of the procedure presented by Wilkie et al [20, 21] for MFC actuators. The design and construction process is outlined in figure 17. The first step consisted of designing the desired interdigitated electrode pattern using a suitable CAD application. Once the design was completed, the electrode pattern was printed on a copper-clad kapton film (Pyralux LF7062R) using photolithography (MetroCircuits Inc). The devices used in this study had an electrode finger width of $0.1 \mathrm{~mm}$ and an electrode finger spacing of $0.5 \mathrm{~mm}$, both parameters consistent with those used in NASA-standard MFCs. PZT-5A piezoceramic rings with $0.2 \mathrm{~mm}$ thickness (EBL Products) were diced into wedgeshaped fibers with a cut angle of $2^{\circ}$ (American Dicing), so that a fiber width of $0.36 \mathrm{~mm}$ was achieved at the inner radius. This value corresponds to the width of prismatic piezoceramic fibers used in typical APT devices [47]. The fibers and electrodes were bonded using an epoxy adhesive system (Hysol Loctite E-120HP).

The transducers were cured in an autoclave, while the standard manufacturing procedure employed a vacuum hot press. Consequently, the cure cycles presented by Wilkie et al [21] were used as a starting point, and fine tuned for the autoclave cure. Each actuator was cured for $2 \mathrm{~h}$ in a vacuum $(-28.2$ in $\mathrm{Hg})$ bag at $100 \mathrm{psi}(689.5 \mathrm{kPa})$ and $250^{\circ} \mathrm{F}\left(121^{\circ} \mathrm{C}\right)$. This cure time and temperature have been previously shown to be sufficient for full actuator cure based on experimentally-calibrated cure kinetics models [20]. A new set of poling parameters was also employed which resulted in 

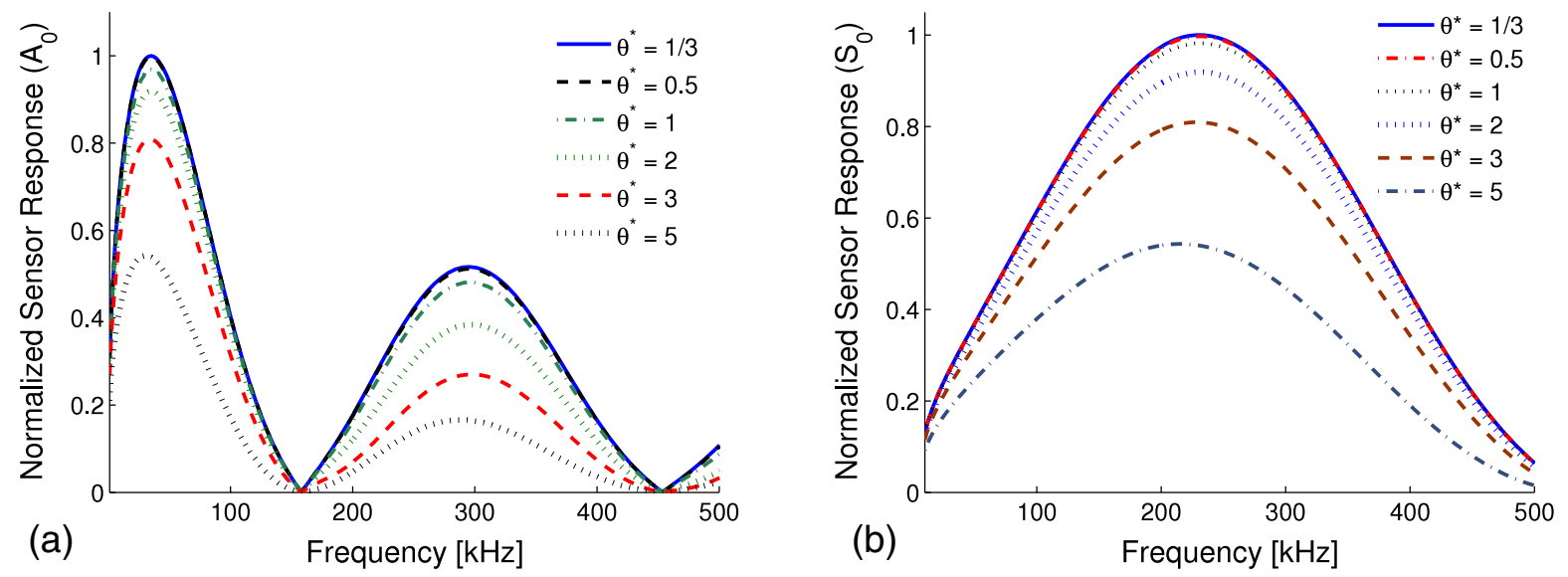

Figure 16. Effect of azimuthal dimension on rectangular APT sensor response. The baseline case was $\Delta r=0.005 \mathrm{~m}, r^{*}=1$. (a) $\mathrm{A}_{0}$ mode. (b) $\mathrm{S}_{0}$ mode.

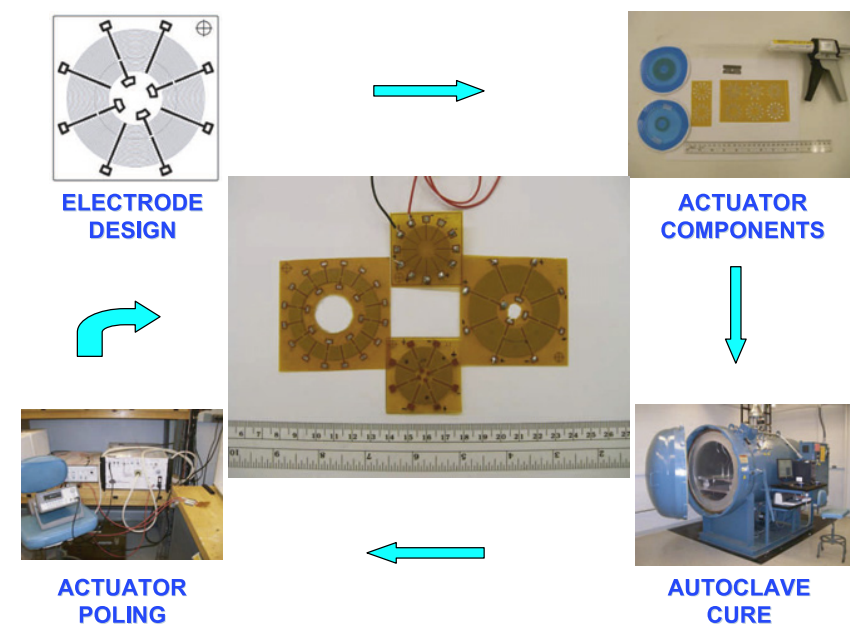

Figure 17. CLoVER transducer development stages.

transducers with performance levels similar to those of NASAstandard MFCs. Further details of the manufacturing and characterization procedures can be found in separate works by the authors $[33,35]$.

\subsection{Sensor-based experiments}

In this section, piezoelectric sensors are employed to verify the accuracy of the theoretical solution in the frequency domain. This method is restricted to single-point measurements, but it is able to provide information on the performance of the transducer over a wide frequency range. This is a critical component of the solution as it enables a transducer designer to determine the transducer size that would induce maximum displacements at a given frequency. This is a key aspect in the transducer design as the excitation frequency is directly related to the damage type to be detected.

7.2.1. Experimental setup. In order to verify the GW excitation model presented in sections 4 and 6 , a $3.2 \mathrm{~mm}-$ thick square aluminum 5005 plate was used in the experimental tests. The plate thickness was selected so that the effect of the actuator's dynamics on the substrate system could be neglected. Past studies have shown successful results employing a similar arrangement [13]. The plate had a side length of $0.7 \mathrm{~m}$. In an effort to excite pure symmetric and antisymmetric modes over a wide frequency range, one CLoVER transducer was bonded on each surface of the plate at its geometric center. The sectors used had a radial dimension $\Delta r=1.0 \mathrm{~cm}\left(R_{\mathrm{O}}=2.5 \mathrm{~cm}, R_{\mathrm{I}}=1.5 \mathrm{~cm}\right)$ and an azimuthal span $\Delta \theta=45^{\circ}$. The symmetric mode was obtained by exciting both actuators in phase, while the antisymmetric mode resulted from exciting them out of phase. The induced wave field was recorded using a square piezoceramic sensor with a side length of $11.6 \mathrm{~mm}$ and a thickness of $0.3 \mathrm{~mm}$. The sensor was located along the transducer's centerline at a radial distance of $76.3 \mathrm{~mm}$. This position was selected based on the plate's dimension, so that boundary reflections were avoided, thereby satisfying the infinite plate assumption. The actuators and the sensor were bonded by applying a thin layer of Epotek 301 bonding agent and allowing it to cure for $24 \mathrm{~h}$. The actuator and sensor arrangement used is illustrated in figure 18(a). Each actuator was excited with a 3.5-cycle Hann-modulated toneburst signal using an arbitrary waveform generator (Agilent 33220A). A digital oscilloscope (Agilent Infinium 54831DSO) was used to monitor and acquire the voltage signal from the sensor, which was averaged over 64 samples at a sampling rate of 10 million samples per second. The overall set up is illustrated in figure 18(b).

7.2.2. Results and discussion. The sensor frequency response was measured experimentally and simulated using the theoretical model in section 6.1. A sample of these results is shown in figure 19, which shows the time history predicted by the model is in good agreement with the data recorded from the piezoelectric sensor. The material properties used for the aluminum alloy in the computations are summarized in table 3 . The figure illustrates the results for the $\mathrm{A}_{0}$ mode at $65 \mathrm{kHz}$. It can be noted that the amplitude and time of arrival of each peak are very well captured by the model, as the error in the time of arrival is less than $2 \%$ while the largest amplitude difference 


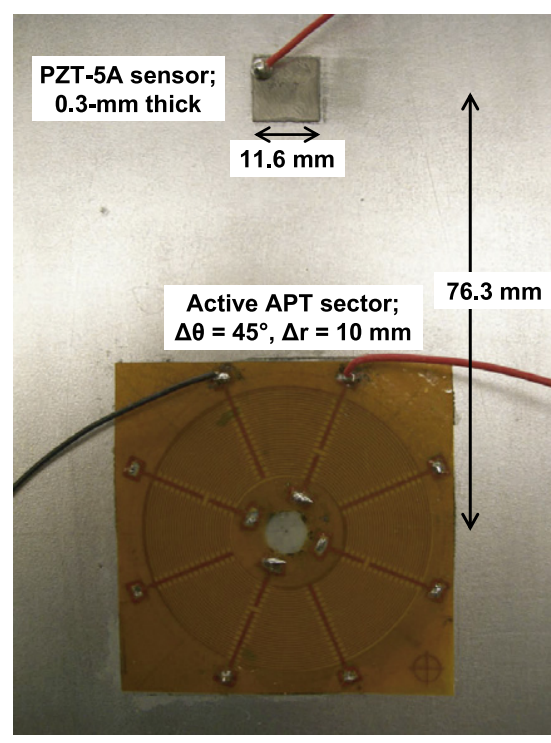

(a)

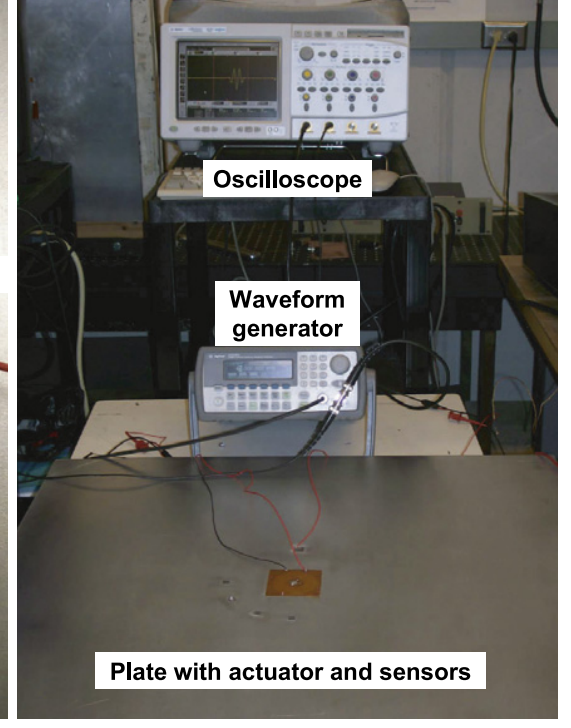

(b)

Figure 18. Experimental setup: (a) CLoVER actuator and sensor detail; (b) overall arrangement.

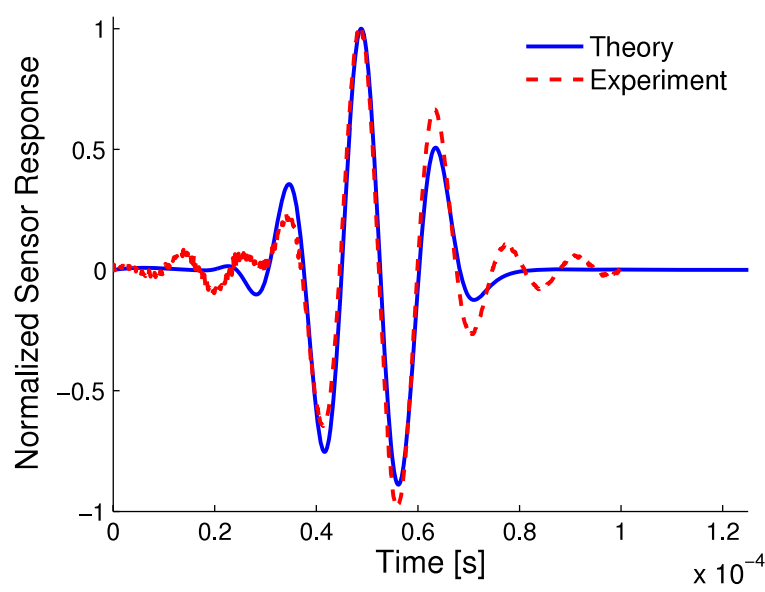

Figure 19. Time history comparison for $\mathrm{A}_{0}$ mode at $65 \mathrm{kHz}$.

(which occurs for the final secondary peak) is less than $8 \%$ of the peak-to-peak amplitude of the pulse.

Several time histories, similar to that presented in figure 19, were recorded over different frequencies for both the symmetric and antisymmetric modes. These results are summarized in figure 20 where each data set has been normalized by its maximum value. The experimental data points represent average values, while the error bars represent three times the peak-to-peak standard deviation. This uncertainty was found by taking 64 averages at each frequency. It can be seen that there is good agreement between the theoretical prediction and the experimental data, especially for the antisymmetric mode where the differences between the two results are within the error bars of the experimental measurements for most of the points. It can also be appreciated that there is good correlation between both results for the symmetric mode until a frequency of approximately $275 \mathrm{kHz}$. Beyond this frequency, the EMI generated by the CLoVER sector is very strong and its interaction with the excitation
Table 3. Aluminum substrate material properties.

\begin{tabular}{ll}
\hline Property & Value \\
\hline$E(\mathrm{GPa})$ & 68.9 \\
$v$ & 0.33 \\
$\rho\left(\mathrm{kg} \mathrm{m}^{-3}\right)$ & 2700 \\
\hline
\end{tabular}

pulse is very significant. Consequently, the peak-to-peak amplitude of the response recorded had to be manually measured according to the expected time of arrival of the pulse, calculated based on its group velocity at each frequency. The EMI results from the rapidly changing electric currents flowing through the interdigitated electrode fingers of the transducer. An important area for further development is the shielding of the transducer and sensor to this type of interference.

\subsection{Laser vibrometer experiments}

In this section, the non-contact technique of laser vibrometry is used to investigate the GW field induced by a CLoVER sector. This method is an important complement to the sensorbased experiments presented in the previous section as it is able to provide information on the spatial variations of the $\mathrm{GW}$ field. The section begins with a brief overview of the operating principles of the scanning laser vibrometer and a description of the experimental setup used. A comprehensive set of results are subsequently presented to verify the spatial and temporal accuracy of the theoretical solution.

7.3.1. Setup and laser vibrometer operation. The key instrument used in these studies was a Polytec PSV-400 scanning laser vibrometer. This system is composed of the PSV-I-400 scanning head, the OFV-5000 controller, the PSVE-401 junction box, and a data management system. The light source used in the PSV-400 is a helium neon laser that 

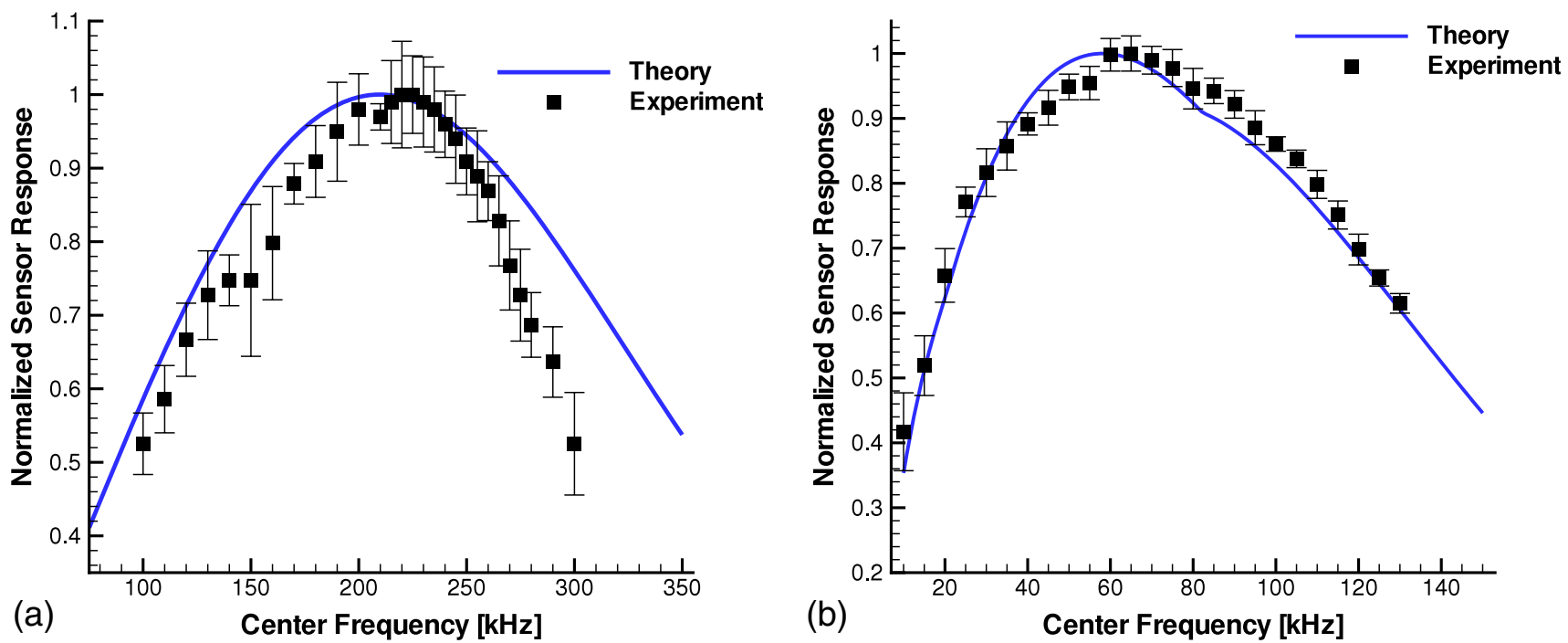

Figure 20. Comparison between theoretical and experimental frequency response results. (a) $S_{0}$ mode, (b) $A_{0}$ mode.

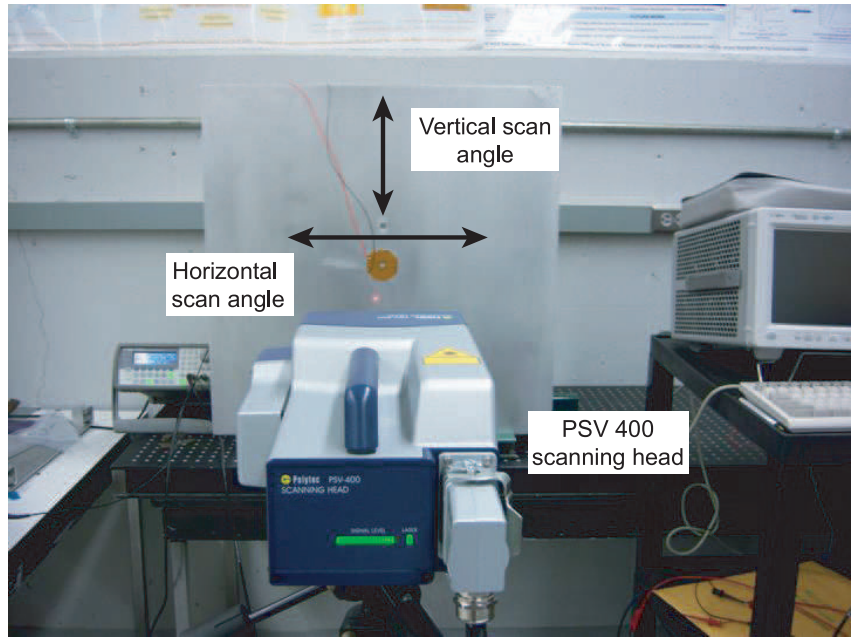

(a)

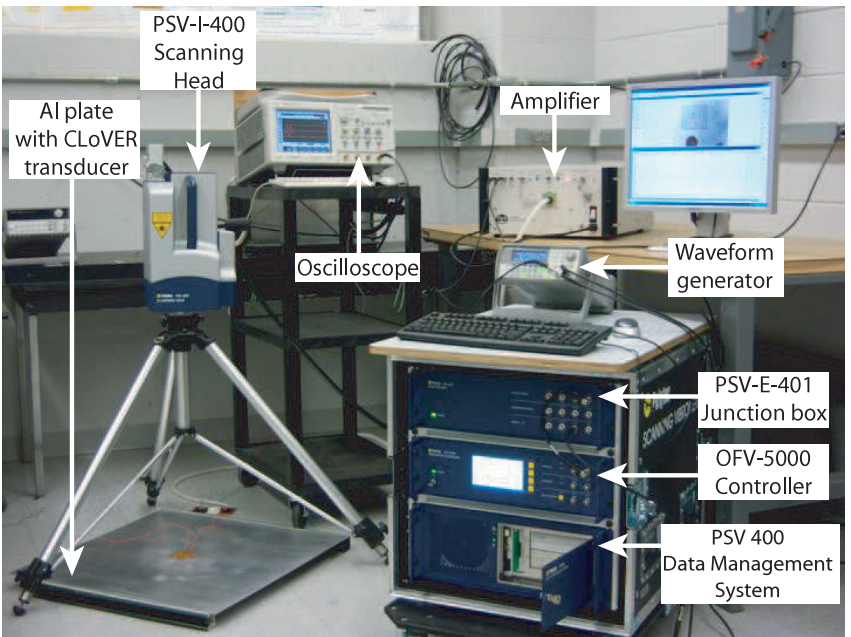

(b)

Figure 21. (a) Definition of horizontal and vertical scan angles; (b) experimental setup used in laser vibrometer tests.

provides a linear polarized beam [48]. The vibrometer system is able to measure the out-of-plane velocities by measuring the difference in path lengths between a reference beam and an object beam that is backscattered from the surface under inspection. The PSV-I-400 scanning head houses a high sensitivity vibrometer sensor, a high-precision scan unit, and a color video camera used to manipulate the laser and scan points in the PSV software. The OFV-5000 controller is able to decode the interference signal of the object and reference beams, while the PSV-E-401 junction box provides an interface for the scanning head, controller, and data management system as well as several input channels for triggering and signal generator output. Finally, the data management system houses the PSV software used to operate the laser vibrometer.

The scanning head was supported using a tripod and maintained at a distance of $915 \mathrm{~mm}$ from the scanning surface for most of the experimental tests conducted. This distance was selected to operate at a laser visibility maximum [48].
An important consideration when taking laser vibrometer measurements is that the scanning angles be small enough so that contributions from the in-plane components remain negligible. The horizontal and vertical scanning angles are defined in figure 21(a). Furthermore, large scanning angles $\left(\sim 10^{\circ}\right)$ are expected to reduce the signal-to-noise ratio. The work of Leong et al [29] provided important insight into this issue. They showed that the vertical scan angle should be kept below $4^{\circ}$ in order to maintain a consistent signal to noise ratio, while the amplitudes of the displacements measured were mostly insensitive to variations in the horizontal scan angle. Consequently, the plate under inspection was arranged so that the vertical scan angle did not exceed the $4^{\circ}$ limit.

The test specimen consisted of the same isotropic plate and CLoVER transducer arrangement described in section 7.2. The surface to be scanned was lightly sanded using 120 grade sand paper to enhance its backscattering behavior. As the laser vibrometer is primarily sensitive to the out-of-plane 


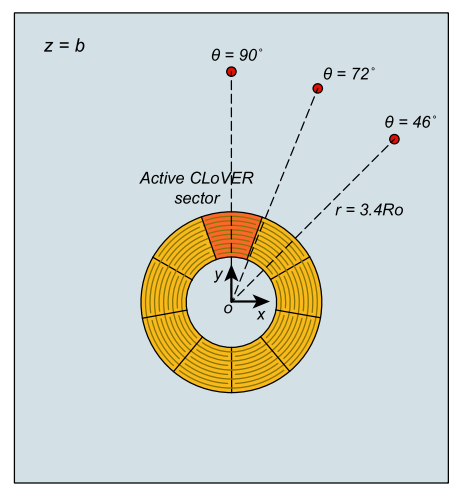

(a)

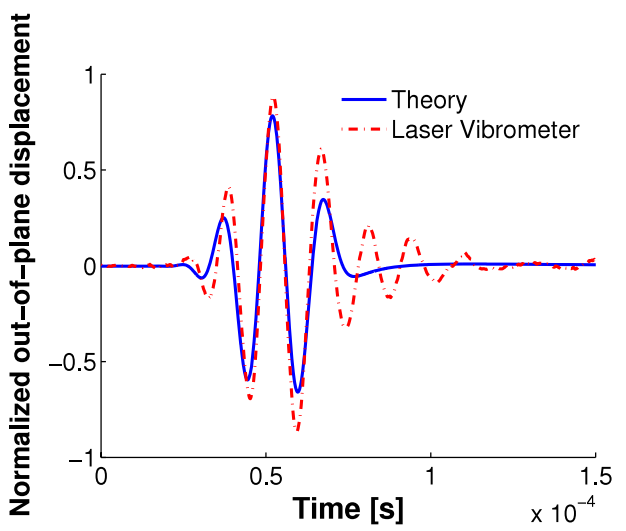

(c)

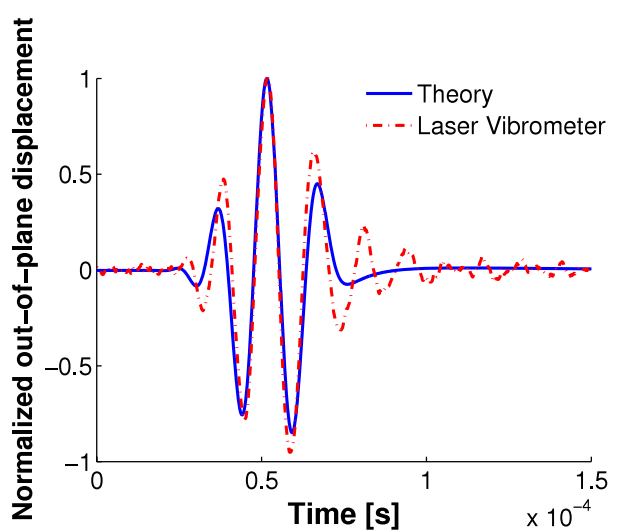

(b)

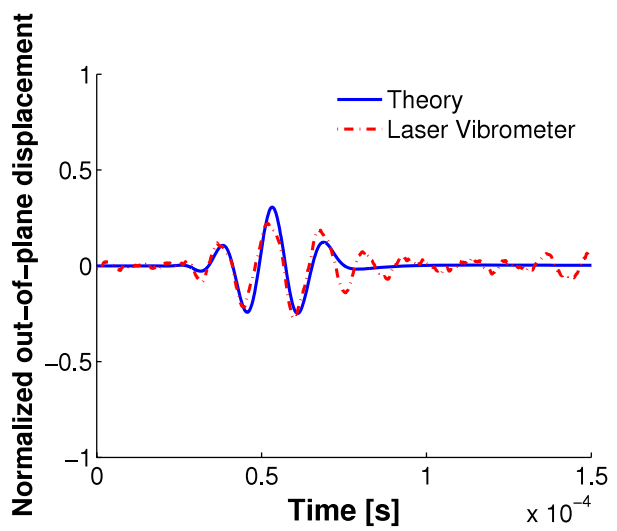

(d)

Figure 22. (a) Schematic illustrating distribution of measurement points; time history comparison at $r=3.2 R_{0}$ and: (b) $\theta=90^{\circ}$; (c) $\theta=72^{\circ}$; (d) $\theta=46^{\circ}$.

velocities, the center frequency of the excitation pulse used was maintained at $65 \mathrm{kHz}$. It was shown earlier through sensor response experiments that the $\mathrm{A}_{0}$ mode is maximized near this frequency. This mode was selected as it is well known that it has a predominantly out-of-plane component. In this case, the excitation pulse was amplified using a Trek PZD2000 high power amplifier which provided a fixed gain of 200. The input voltage used in this set of tests was higher than in the sensor tests to obtain a high signal-to-noise ratio in the laser vibrometer, and varied from 200 to $800 \mathrm{~V}_{\mathrm{p}-\mathrm{p}}$. The sampling frequency used in the data acquisition system was $5.12 \mathrm{MHz}$. The overall experimental setup is illustrated in figure 21(b).

7.3.2. Results and discussion. The first set of tests consisted of evaluating the time history performance of the solution. Three points were selected at a fixed distance of $3.4 R_{\mathrm{O}}$ and various azimuthal locations, as shown in figure 22(a). The time of arrival is close for both solutions (within 5\%) as shown in figures 22(b)-(d). The shape of the toneburst signal is also well captured although there are some differences in the relative amplitude of both the main and secondary peaks. The largest of these differences occurs for the time history recorded at $72^{\circ}$ and corresponds to approximately $30 \%$ of the peak-to-peak amplitude of the excitation pulse. As previously mentioned, the excitation voltage used in these experiments was significantly larger than that used in the sensor-based experiments, partially to obtain a good signal to noise ratio. A larger voltage input generates larger strains in the transducer, and it is possible that this results in a more pronounced contribution from the transducer dynamics which have been neglected in the theoretical solution. It should be noted that there are additional reasons for selecting a higher excitation voltage in this set of experiments. First, in this case only one transducer is being used in contrast to the two used in the previous experiments. This was done because in preliminary testing it was found that due to the sensitivity of the laser beam, minor misalignments in the placement of the transducers ( $\sim 2 \mathrm{~mm}$ ) significantly affected the shape of the propagating pulse. It is likely that the sensor is not as sensitive to these differences as it is primarily excited by the weaker (in the $\mathrm{A}_{0}$ case) in-plane displacements. In addition, the piezoceramic fibers used in the CLoVER transducer are relatively thin. Finally, the antisymmetric mode is being excited using shear tractions in the plane of the substrate. It has been previously reported [49] that, due to this mode's strong out-of-plane component, transducers that apply surface tractions normal to the surface are preferred for $\mathrm{A}_{0}$ excitation when employing a single device. 


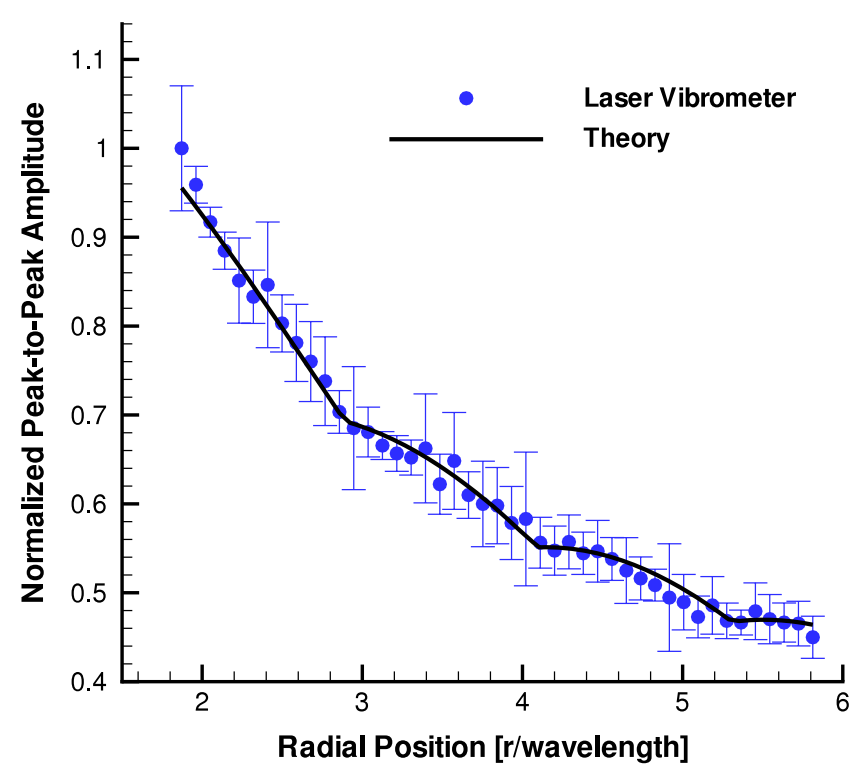

Figure 23. Comparison between theoretical and laser vibrometer results for peak-to-peak amplitude.

The second set of tests consisted of evaluating the decay in the propagating pulse amplitude with radial distance. It is important to accurately capture this parameter in the theoretical solution as it is key in determining the inspection distance that can be achieved with the transducer. In this case, several radial positions oriented along the centerline of a CLoVER sector were selected and the laser was used to measure the time history. The peak-to-peak amplitude obtained from these measurements were then compared to similar results obtained using the theoretical model. This result is shown in figure 23, where the results have been normalized by the mean peak-to-peak amplitude in each data set. As in previous cases, the error bars represent three times the standard deviation. This uncertainty was found by repeating each measurement three times, collecting 64 averages in each test. The figure shows that the experimental and theoretical results are in excellent agreement, with the differences between them staying within the experimental uncertainty for all the measurement points. Note that neither the theoretical model nor the numerical simulations have accounted for material damping, and therefore the amplitude attenuation observed in the results in figure 23 are due to geometric attenuation only. The good agreement between both results indicates that accounting for material damping is not necessary when working with linearly elastic isotropic materials.

Another important consideration is the azimuthal distribution of the induced GW field. This parameter indicates the directionality achieved by a CLoVER sector. As in the radial decay study, the peak-to-peak displacement amplitude was measured at several azimuthal and radial locations as shown in figure $24(\mathrm{a})$. In this case, a complete $360^{\circ}$ range was scanned using a grid consisting of 60 azimuthal points. In addition, three radial locations were chosen to investigate the evolution of the azimuthal distribution with radial distance. The measurements were repeated three different times with each set consisting of 64 averages. The displacement field was calculated theoretically for the same radial locations using a finer grid of azimuthal points. These results are compared in several polar plots shown in figures 24(b)-(d). In these figures, the transducer centerline coincides with the $90^{\circ}$ direction, and the peak-to-peak amplitude is represented by the radial distance from the origin for each azimuthal location. The uncertainty for each point is represented by two finer lines surrounding the experimental points which correspond to three times the standard deviation.

The results show that the azimuthal distribution is captured well by the model. The regions where the largest disagreements are observed correspond to normal directions from the transducer centerline where the GW field is smallest, which in turn decreases the signal-to-noise ratio in the laser vibrometer resulting in large standard deviations. Some differences are also observed for the wave field induced in the opposite direction, i.e., towards the $270^{\circ}$ direction, especially for larger radial distances. The largest error in this case is in the order of $23 \%$ and it occurs for the farthest radial position tested, at an azimuthal location of approximately $300^{\circ}$. It is likely that this disagreement is partly due to the presence of additional CLoVER sectors which are not accounted for in the theoretical solution. These additional transducers introduce concentrated masses which act as very small GW scatterers. As the radial position is increased, a larger portion of the wave field interacts with the additional sectors, which may explain the increasing difference with larger distance. It can also be appreciated from the figures that as the radial position is increased, the main displacement lobe becomes wider, which is consistent with the expectation that in the far field the wavefront would tend to a uniform circular front [38].

The final set of tests consisted of a full-field scan to visually compare the temporal and spatial evolution of the GW field induced by a CLoVER sector. The scanning grid used in this case consisted of 25 radial points distributed over a $7 \mathrm{~cm}$ range, which provided approximately three wavelengths with seven points per wavelength. A complete $360^{\circ}$ range was covered using an azimuthal grid consisting of 40 points. The starting radial position for the measurements was selected at $1 \mathrm{~cm}$ from the radial edge of the CLoVER sector to avoid scanning over pieces of kapton film that remain attached to house the electrode bus of the transducer. Similarly, the radial positions scanned excluded points near the origin as a piezoelectric sensor was bonded in this area. These results are summarized in figures 25 through 27 , which show very good correlation between the theoretical model and the experiment. The directionality of the transducer is clearly visible, as the induced displacements remain primarily within the azimuthal span of the CLoVER sector. The results presented in the previous sections have shown the accuracy of the threedimensional elasticity solution in describing the GW field induced by a CLoVER sector in isotropic structures. The performance of this device in damage detection and location has also been explored in separate studies $[33,50]$ by the authors, where it has been shown that its directionality and geometry allow it to accurately detect simulated defects in metallic and composite plate-like structures. 


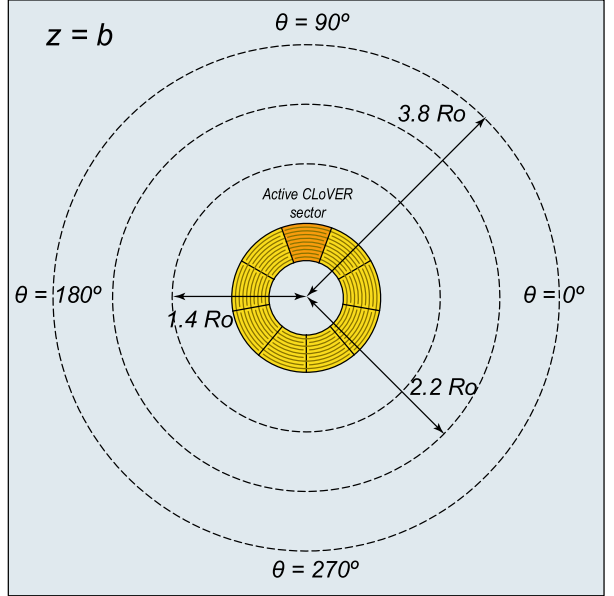

(a)

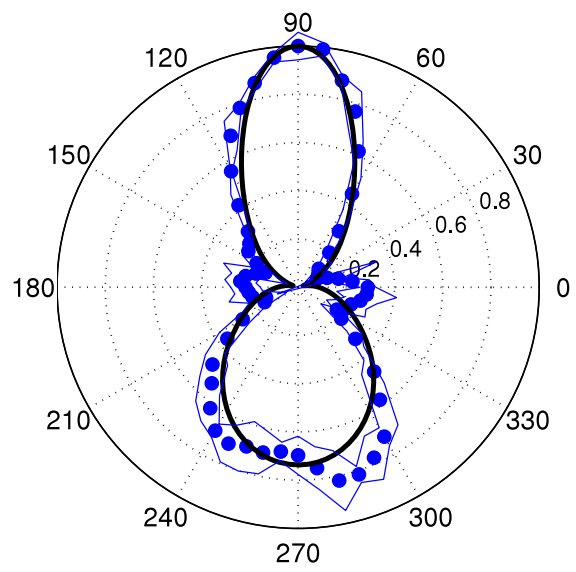

(c)

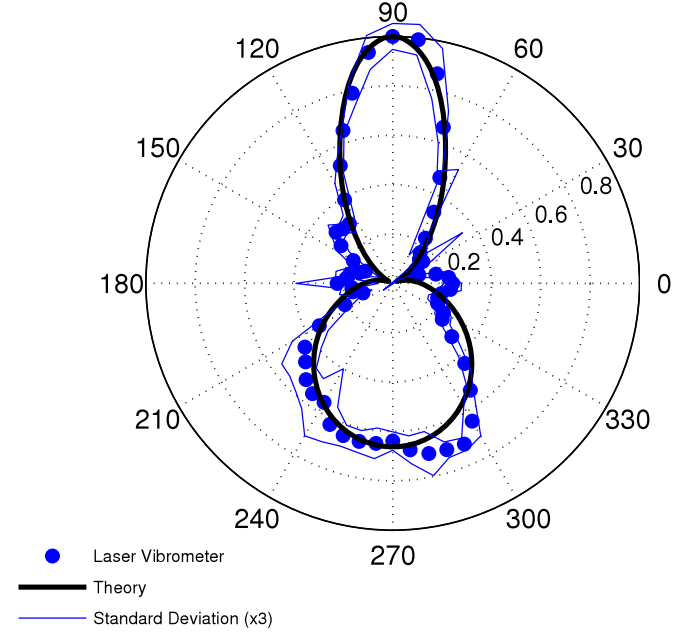

(b)

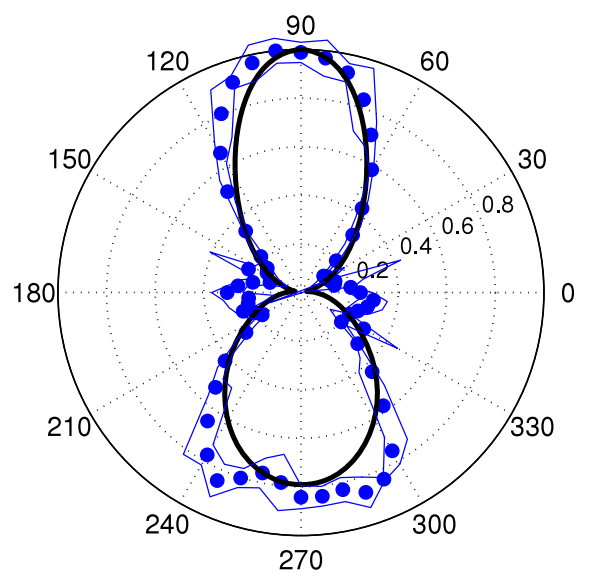

(d)

Figure 24. (a) Schematic showing location of the scan points used in azimuthal comparison tests; comparison of peak-to-peak amplitudes at: (b) $r=1.4 R_{\mathrm{O}}$; (c) $r=2.2 R_{\mathrm{O}}$; (d) $r=3.8 R_{\mathrm{O}}$.

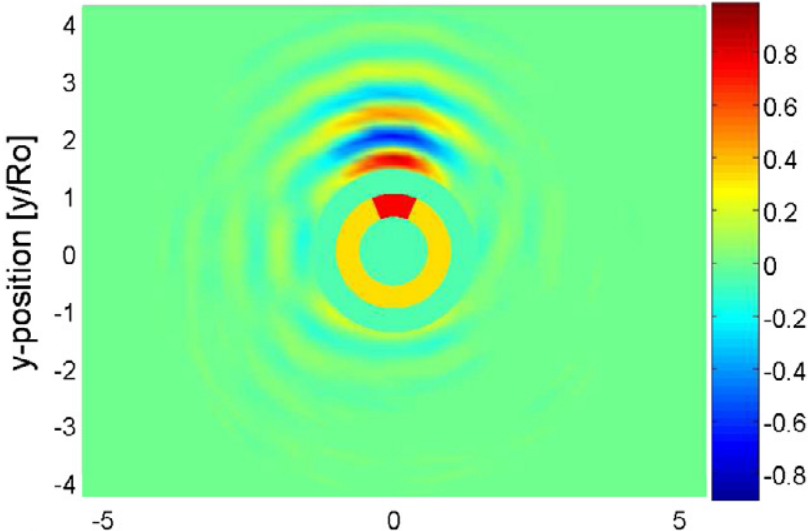

(a)

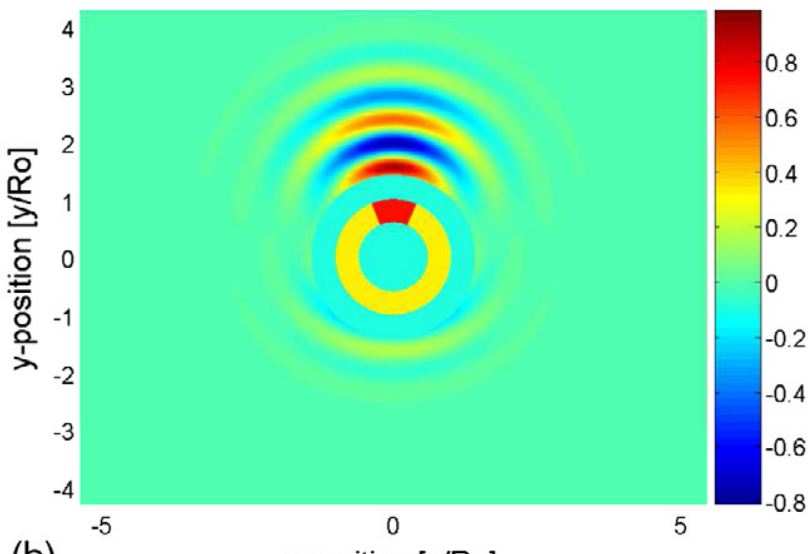

(b)

x-position [x/Ro]

Figure 25. Full-field comparison between laser vibrometer and theoretical solution at time $t=35 \mu \mathrm{s}$. (a) Experiment. (b) Theory.

\section{Summary and conclusions}

The guided wave (GW) field excited by a surface-bonded, wedge-shaped anisotropic piezocomposite transducer (APT) was investigated in this paper. This study supports the development of the composite long-range variable-direction emitting radar (CLoVER) transducer. This novel device has been introduced by the authors as an alternative concept for 

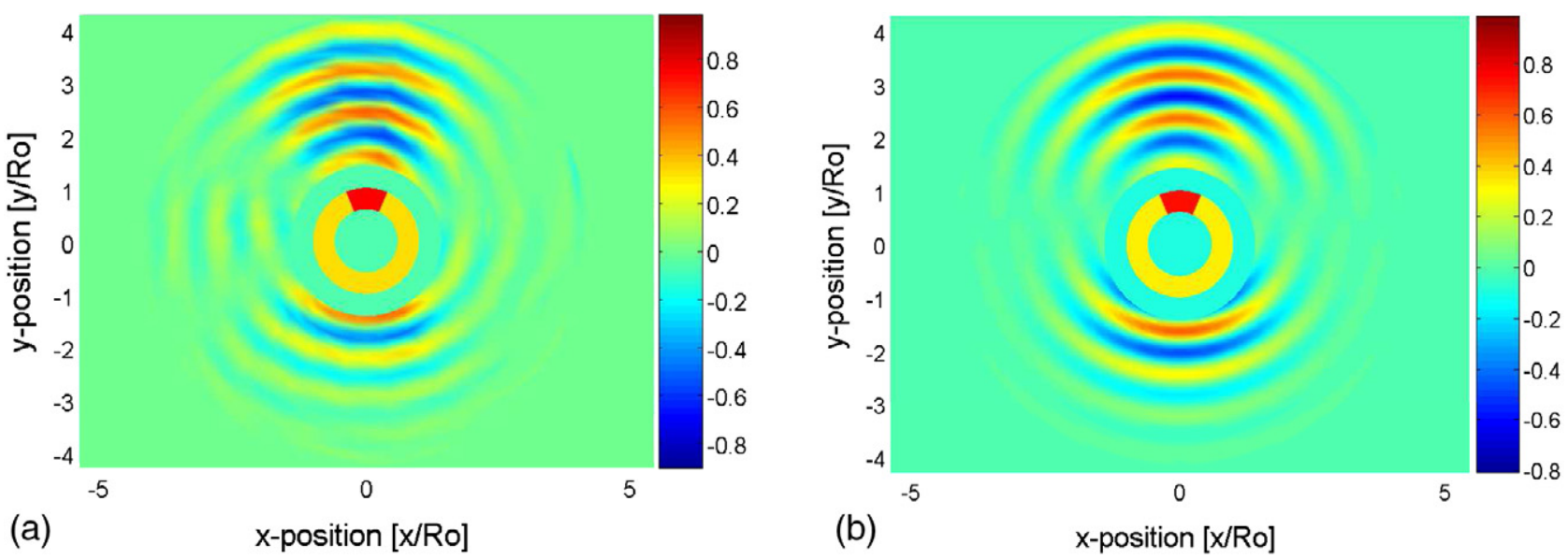

Figure 26. Full-field comparison between laser vibrometer and theoretical solution at time $t=50 \mu$ s. (a) Experiment. (b) Theory.
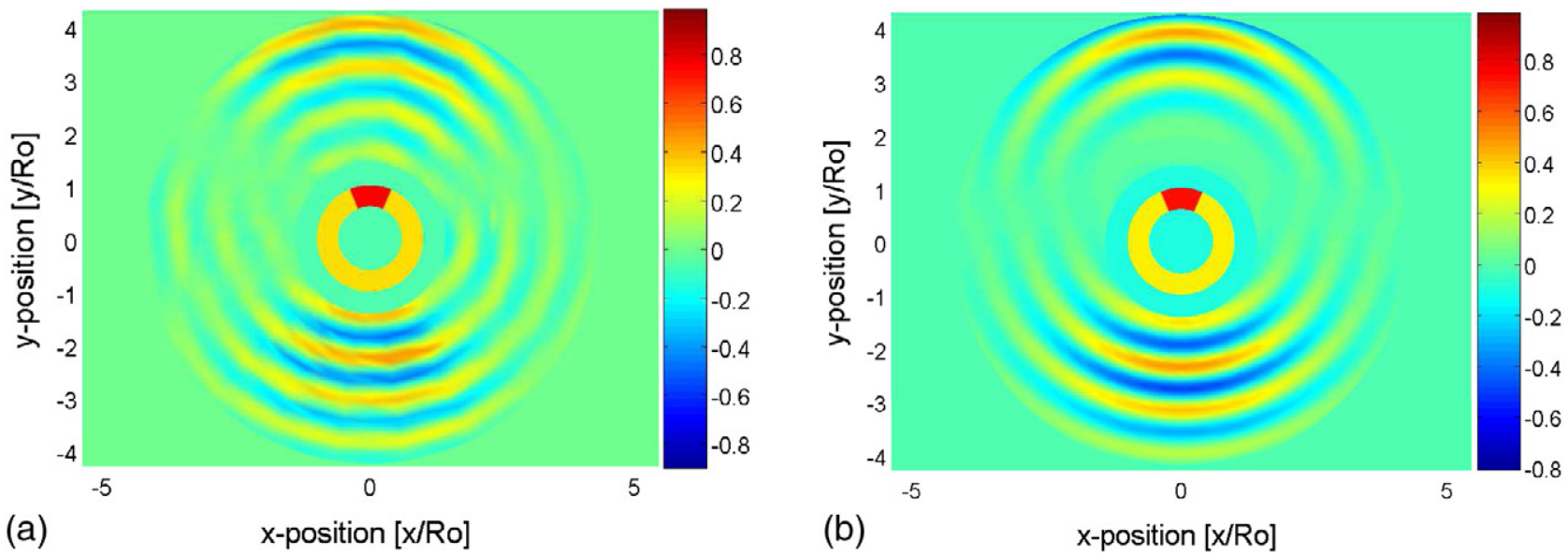

Figure 27. Full-field comparison between laser vibrometer and theoretical solution at time $t=65 \mu \mathrm{s}$. (a) Experiment. (b) Theory.

damage interrogation and GW excitation in structural health monitoring (SHM) systems. This transducer has an overall ring geometry, but is composed of wedge-shaped APT sectors that can be individually excited to interrogate the structure along a particular direction from a central location. Some of the advantages offered by this new design were briefly discussed. The theoretical analysis considered uncoupled dynamics for the actuator and substrate, and their interaction was modeled as surface tractions along the actuator's edges. Under these assumptions, a 3D elasticity boundary value problem was formulated, and its solution yielded theoretical expressions for the displacement field. Results from three-dimensional finite element simulations demonstrated the accuracy of the theoretical result as a solution to the elasticity problem. The sensor response of a uniform rectangular piezoelectric wafer and a wedge-shaped APT under the GW field excited by a wedge-shaped APT was also investigated. This analysis was based on modeling the actuators as capacitors. The rectangular piezoelectric sensor was considered as a parallel plate capacitor, while the APT was considered as a combination of parallel plate capacitors. It was found that the radial dimension of the APT sensor significantly affects the response in frequency, while the azimuthal span only affects the response amplitude. The overall trend that smaller sensors produce enhanced response was confirmed. A combination of sensor-based and laser vibrometer-based experiments were conducted to verify the performance of the solution in representing the actual physical system. A comprehensive set of results was presented and the ability of the solution to capture the experimental results in the time, frequency, and space domains was demonstrated.

\section{Acknowledgments}

This work was partly supported by the Air Force Office of Scientific Research under grants FA9550-06-1-0071 and FA9550-07-1-0522 with Dr Victor Giurgiutiu as the technical monitor. This work was also supported by the NASA Constellation University Institutes Project under grant Z634001, with Ms Claudia Meyer as the project manager. Technical discussions with Dr Ajay Raghavan (The University of Michigan, presently at Metis Design Corporation) at various stages of this work are gratefully acknowledged. 


\section{Appendix}

The column vector of coefficients, $\boldsymbol{\Delta}$, first introduced in equation (41), is defined through:

$$
\Delta=\left\{\begin{array}{c}
\varrho_{-2-k}^{(1)}-\varrho_{-k}^{(2)}-\varrho_{2-k}^{(3)} \\
\varsigma_{-2-k}^{(1)}-\varsigma_{-k}^{(2)}-\varsigma_{2-k}^{(3)} \\
\bar{\delta}_{1-k}^{(1)}+\bar{\delta}_{-1-k}^{(2)}
\end{array}\right\}
$$

where the individual components have the following definitions:

$$
\begin{aligned}
& \varrho_{k}^{(1)}= {\left[c_{k}^{(2)} \gamma_{5}^{(1)}-c_{k}^{(1)} \gamma_{4}^{(1)}\right](-\mathrm{i})^{k} \chi_{k} } \\
& \varrho_{k}^{(2)}=c_{k}^{(1)} \gamma_{3}^{(1)}(-\mathrm{i})^{k} \chi_{k} \\
& \varrho_{k}^{(3)}=\left[c_{k}^{(1)} \gamma_{4}^{(1)}+c_{k}^{(2)} \gamma_{5}^{(1)}\right](-\mathrm{i})^{k} \chi_{k} \\
& \varsigma_{k}^{(1)}=\left[c_{k}^{(1)} \gamma_{1}^{(2)}-c_{k}^{(2)} \gamma_{5}^{(2)}\right](-\mathrm{i})^{k} \chi_{k} \\
& \zeta_{k}^{(2)}=c_{k}^{(2)} \gamma_{4}^{(2)}(-\mathrm{i})^{k} \chi_{k} \\
& \varsigma_{k}^{(3)}=\left[c_{k}^{(1)} \gamma_{1}^{(2)}+c_{k}^{(2)} \gamma_{5}^{(2)}\right](-\mathrm{i})^{k} \chi_{k} \\
& \bar{\delta}_{k}^{(1)}=\frac{1}{2}\left(c_{k}^{(1)}+\mathrm{i} c_{k}^{(2)}\right)(-\mathrm{i})^{k} \chi_{k} \\
& \bar{\delta}_{k}^{(2)}=\frac{1}{2}\left(c_{k}^{(1)}-\mathrm{i} c_{k}^{(2)}\right)(-\mathrm{i})^{k} \chi_{k} .
\end{aligned}
$$

Similarly, the column vector $\boldsymbol{\Lambda}$ is defined as:

$$
\boldsymbol{\Lambda}=\left\{\begin{array}{c}
\eta_{-2-k}^{(1)}-\eta_{-k}^{(2)}-\eta_{2-k}^{(3)} \\
\kappa_{-2-k}^{(1)}-\kappa_{-k}^{(2)}-\kappa_{2-k}^{(3)} \\
\bar{v}_{1-k}^{(1)}+\bar{v}_{-1-k}^{(2)}
\end{array}\right\}
$$

where the individual components have the following definitions:

$$
\begin{aligned}
\eta_{k}^{(1)} & =\left[c_{k}^{(2)} \gamma_{5}^{(1)}-c_{k}^{(1)} \gamma_{4}^{(1)}\right]\left[R_{\mathrm{O}} H_{k}^{(2)}\left(\xi_{\mathrm{A}} r\right)\right. \\
& \left.-R_{\mathrm{I}} H_{k}^{(2)}\left(\xi_{\mathrm{A}} r\right)\right](-\mathrm{i})^{k} \\
\eta_{k}^{(2)} & =c_{k}^{(1)} \gamma_{3}^{(1)}\left[R_{\mathrm{O}} H_{k}^{(2)}\left(\xi_{\mathrm{A}} r\right)-R_{\mathrm{I}} H_{k}^{(2)}\left(\xi_{\mathrm{A}} r\right)\right](-\mathrm{i})^{k} \\
\eta_{k}^{(3)} & =\left[c_{k}^{(1)} \gamma_{4}^{(1)}+c_{k}^{(2)} \gamma_{5}^{(1)}\right]\left[R_{\mathrm{O}} H_{k}^{(2)}\left(\xi_{\mathrm{A}} r\right)\right. \\
& \left.-R_{\mathrm{I}} H_{k}^{(2)}\left(\xi_{\mathrm{A}} r\right)\right](-\mathrm{i})^{k} \\
\kappa_{k}^{(1)} & =\left[c_{k}^{(1)} \gamma_{1}^{(2)}-c_{k}^{(2)} \gamma_{5}^{(2)}\right]\left[R_{\mathrm{O}} H_{k}^{(2)}\left(\xi_{\mathrm{A}} r\right)\right. \\
& \left.-R_{\mathrm{I}} H_{k}^{(2)}\left(\xi_{\mathrm{A}} r\right)\right](-\mathrm{i})^{k} \\
\kappa_{k}^{(2)} & =c_{k}^{(2)} \gamma_{4}^{(2)}\left[R_{\mathrm{O}} H_{k}^{(2)}\left(\xi_{\mathrm{A}} r\right)-R_{\mathrm{I}} H_{k}^{(2)}\left(\xi_{\mathrm{A}} r\right)\right](-\mathrm{i})^{k} \\
\kappa_{k}^{(3)} & =\left[c_{k}^{(1)} \gamma_{1}^{(2)}+c_{k}^{(2)} \gamma_{5}^{(2)}\right]\left[R_{\mathrm{O}} H_{k}^{(2)}\left(\xi_{\mathrm{A}} r\right)\right. \\
& \left.-R_{\mathrm{I}} H_{k}^{(2)}\left(\xi_{\mathrm{A}} r\right)\right](-\mathrm{i})^{k} \\
\bar{v}_{k}^{(1)} & =\frac{1}{2}\left(c_{k}^{(1)}+\mathrm{i} c_{k}^{(2)}\right)\left[R_{\mathrm{O}} H_{k}^{(2)}\left(\xi_{\mathrm{A}} r\right)\right. \\
& \left.-R_{\mathrm{I}} H_{k}^{(2)}\left(\xi_{\mathrm{A}} r\right)\right](-\mathrm{i})^{k} \\
\bar{v}_{k}^{(2)} & =\frac{1}{2}\left(c_{k}^{(1)}-\mathrm{i} c_{k}^{(2)}\right)\left[R_{\mathrm{O}} H_{k}^{(2)}\left(\xi_{\mathrm{A}} r\right)\right. \\
& \left.-R_{\mathrm{I}} H_{k}^{(2)}\left(\xi_{\mathrm{A}} r\right)\right](-\mathrm{i})^{k} .
\end{aligned}
$$

The column vector $\tilde{\Upsilon}$ is defined as:

$$
\tilde{\boldsymbol{\Upsilon}}=\left\{\begin{array}{c}
\tilde{\rho}_{-2-k}^{(1)}-\tilde{\rho}_{-k}^{(2)}-\tilde{\rho}_{2-k}^{(3)} \\
\tilde{\psi}_{-2-k}^{(1)}-\tilde{\psi}_{-k}^{(2)}-\tilde{\psi}_{2-k}^{(3)} \\
\tilde{v}_{1-k}^{(1)}+\tilde{v}_{-1-k}^{(2)}
\end{array}\right\}
$$

where the individual components are given by:

$$
\begin{gathered}
\tilde{\rho}_{k}^{(1)}=\left[c_{k}^{(2)} \gamma_{5}^{(1)}-c_{k}^{(1)} \gamma_{4}^{(1)}\right](-\mathrm{i})^{k} R_{\mathrm{O}} H_{k}^{(2)}\left(\xi_{\mathrm{A}} R_{\mathrm{O}}\right) \\
\tilde{\rho}_{k}^{(2)}=c_{k}^{(1)} \gamma_{3}^{(1)}(-\mathrm{i})^{k} R_{\mathrm{O}} H_{k}^{(2)}\left(\xi_{\mathrm{A}} R_{\mathrm{O}}\right) \\
\tilde{\rho}_{k}^{(2)}=\left[c_{k}^{(1)} \gamma_{4}^{(1)}+c_{k}^{(2)} \gamma_{5}^{(1)}\right](-\mathrm{i})^{k} R_{\mathrm{O}} H_{k}^{(2)}\left(\xi_{\mathrm{A}} R_{\mathrm{O}}\right) \\
\tilde{\psi}_{k}^{(1)}=\left[c_{k}^{(1)} \gamma_{1}^{(2)}-c_{k}^{(2)} \gamma_{5}^{(2)}\right](-\mathrm{i})^{k} R_{\mathrm{O}} H_{k}^{(2)}\left(\xi_{\mathrm{A}} R_{\mathrm{O}}\right) \\
\tilde{\psi}_{k}^{(2)}=c_{k}^{(2)} \gamma_{4}^{(2)}(-\mathrm{i})^{k} R_{\mathrm{O}} H_{k}^{(2)}\left(\xi_{\mathrm{A}} R_{\mathrm{O}}\right) \\
\tilde{\psi}_{k}^{(3)}=\left[c_{k}^{(1)} \gamma_{1}^{(2)}+c_{k}^{(2)} \gamma_{5}^{(2)}\right](-\mathrm{i})^{k} R_{\mathrm{O}} H_{k}^{(2)}\left(\xi_{\mathrm{A}} R_{\mathrm{O}}\right) \\
\tilde{v}_{k}^{(1)}=\frac{1}{2}\left(c_{k}^{(1)}+\mathrm{i} c_{k}^{(2)}\right)(-\mathrm{i})^{k} R_{\mathrm{O}} H_{k}^{(2)}\left(\xi_{\mathrm{A}} R_{\mathrm{O}}\right) \\
\tilde{v}_{k}^{(2)}=\frac{1}{2}\left(c_{k}^{(1)}-\mathrm{i} c_{k}^{(2)}\right)(-\mathrm{i})^{k} R_{\mathrm{O}} H_{k}^{(2)}\left(\xi_{\mathrm{A}} R_{\mathrm{O}}\right) .
\end{gathered}
$$

Finally, the column vector $\Upsilon$ is defined as:

$$
\Upsilon=\left\{\begin{array}{c}
\bar{\rho}_{-2-k}^{(1)}-\bar{\rho}_{-k}^{(2)}-\bar{\rho}_{2-k}^{(3)} \\
\hat{\psi}_{-2-k}^{(1)}-\hat{\psi}_{-k}^{(2)}-\hat{\psi}_{2-k}^{(3)} \\
v_{1-k}^{(1)}+v_{-1-k}^{(2)}
\end{array}\right\}
$$

where the individual components are given by:

$$
\begin{gathered}
\bar{\rho}_{k}^{(1)}=\left[c_{k}^{(2)} \gamma_{5}^{(1)}-c_{k}^{(1)} \gamma_{4}^{(1)}\right](-\mathrm{i})^{k} R_{\mathrm{I}} J_{k}\left(\xi_{\mathrm{A}} R_{\mathrm{I}}\right) \\
\bar{\rho}_{k}^{(2)}=c_{k}^{(1)} \gamma_{3}^{(1)}(-\mathrm{i})^{k} R_{\mathrm{I}} J_{k}\left(\xi_{\mathrm{A}} R_{\mathrm{I}}\right) \\
\bar{\rho}_{k}^{(2)}=\left[c_{k}^{(1)} \gamma_{4}^{(1)}+c_{k}^{(2)} \gamma_{5}^{(1)}\right](-\mathrm{i})^{k} R_{\mathrm{I}} J_{k}\left(\xi_{\mathrm{A}} R_{\mathrm{I}}\right) \\
\hat{\psi}_{k}^{(1)}=\left[c_{k}^{(1)} \gamma_{1}^{(2)}-c_{k}^{(2)} \gamma_{5}^{(2)}\right](-\mathrm{i})^{k} R_{\mathrm{I}} J_{k}\left(\xi_{\mathrm{A}} R_{\mathrm{I}}\right) \\
\hat{\psi}_{k}^{(2)}=c_{k}^{(2)} \gamma_{4}^{(2)}(-\mathrm{i})^{k} R_{\mathrm{I}} J_{k}\left(\xi_{\mathrm{A}} R_{\mathrm{I}}\right) \\
\hat{\psi}_{k}^{(3)}=\left[c_{k}^{(1)} \gamma_{1}^{(2)}+c_{k}^{(2)} \gamma_{5}^{(2)}\right](-\mathrm{i})^{k} R_{\mathrm{I}} J_{k}\left(\xi_{\mathrm{A}} R_{\mathrm{I}}\right) \\
v_{k}^{(1)}=\frac{1}{2}\left(c_{k}^{(1)}+\mathrm{i} c_{k}^{(2)}\right)(-\mathrm{i})^{k} R_{\mathrm{I}} J_{k}\left(\xi_{\mathrm{A}} R_{\mathrm{I}}\right) \\
v_{k}^{(2)}=\frac{1}{2}\left(c_{k}^{(1)}-\mathrm{i} c_{k}^{(2)}\right)(-\mathrm{i})^{k} R_{\mathrm{I}} J_{k}\left(\xi_{\mathrm{A}} R_{\mathrm{I}}\right) .
\end{gathered}
$$

The distinction between symmetric and antisymmetric modes occurs in the definition of the coefficients $\gamma_{j}^{(i)}$. The following equations provide their definition for the antisymmetric case. The coefficients for the symmetric mode are found analogously by interchanging sine and cosine terms whose arguments depend on the substrate half-thickness $b$, and by replacing the antisymmetric wavenumber, $\xi_{\mathrm{A}}$, by its symmetric counterpart, $\xi_{\mathrm{S}}$.

$$
\begin{gathered}
\gamma_{1}^{(1)}=\sin \alpha b \cos \beta b \\
\gamma_{2}^{(1)}=4 \alpha \beta \cos \alpha b \sin \beta b
\end{gathered}
$$$$
\gamma_{3}^{(1)}=\gamma_{1}^{(1)}\left(\frac{\xi_{\mathrm{A}}^{4}}{2}+\beta^{4}-\beta^{2} \frac{\xi_{\mathrm{A}}^{2}}{2}\right)+\gamma_{2}^{(1)} \frac{\xi_{\mathrm{A}}^{2}}{2}
$$$$
\gamma_{4}^{(1)}=\gamma_{1}^{(1)}\left(\frac{3}{4} \xi_{\mathrm{A}}^{2} \beta^{2}-\frac{\xi_{\mathrm{A}}^{4}}{4}\right)-\frac{\gamma_{2}^{(1)}}{2} \xi_{\mathrm{A}}^{2}
$$

$\gamma_{5}^{(1)}=-\mathrm{i} \frac{\xi_{\mathrm{A}}^{2}}{4}\left[\left(\xi_{\mathrm{A}}^{2}-3 \beta^{2}\right) \sin \alpha b \cos \beta b+4 \alpha \beta \cos \alpha b \sin \beta b\right]$ 


$$
\begin{gathered}
\gamma_{1}^{(2)}=-\mathrm{i} \frac{\xi_{\mathrm{A}}^{2}}{4}\left[\left(\xi_{\mathrm{A}}^{2}-3 \beta^{2}\right) \sin \alpha b \cos \beta b+4 \alpha \beta \cos \alpha b \sin \beta b\right] \\
\gamma_{2}^{(2)}=\sin \alpha b \cos \beta b \\
\gamma_{3}^{(2)}=4 \alpha \beta \cos \alpha b \sin \beta b \\
\gamma_{4}^{(2)}=\gamma_{2}^{(2)}\left(\frac{\xi_{\mathrm{A}}^{4}}{2}+\beta^{4}-\beta^{2} \frac{\xi_{\mathrm{A}}^{2}}{2}\right)+\gamma_{3}^{(2)} \frac{\xi_{\mathrm{A}}^{2}}{2} \\
\gamma_{5}^{(2)}=\gamma_{2}^{(2)}\left(\frac{\xi_{\mathrm{A}}^{4}}{4}-\frac{3}{4} \xi_{\mathrm{A}}^{2} \beta^{2}\right)+\frac{\gamma_{3}^{(2)}}{4} \xi_{\mathrm{A}}^{2} .
\end{gathered}
$$

\section{References}

[1] Farrar C R and Worden K 2007 An introduction to structural health monitoring Phil. Trans. R. Soc. A 365 303-15

[2] Worden K and Farrar C R 2007 The fundamental axioms of structural health monitoring Phil. Trans. R. Soc. A 463 1639-64

[3] Raghavan A and Cesnik C E S 2007 Review of guided-wave based structural health monitoring Shock Vib. Dig. 39 91-114

[4] Lamb H 1917 On the waves in an elastic plate Proc. R. Soc. A $93293-312$

[5] Gazis D C 1958 Exact analysis of the plane-strain vibrations of thick-walled hollow cylinders J. Acoust. Soc. Am. 30 786-94

[6] Wilcox P D, Cawley P and Lowe M J S 1998 Acoustic fields from PVDF interdigital transducers IEEE Proc. Sci. Meas. Technol. 145 250-9

[7] Achenbach J D and Xu Y 1999 Wave motion in an isotropic elastic layer generated by a time-harmonic point force of arbitrary direction J. Acoust. Soc. Am. 106 83-90

[8] Lin X and Yuan F G 2001 Diagnostic Lamb waves in an integrated piezoelectric sensor/actuator plate: analytical and experimental results Smart Mater. Struct. 10 907-13

[9] Rose L R F and Wang C H 2003 Mindlin plate theory for damage detection: source solutions J. Acoust. Soc. Am. 116 154-71

[10] Yang S and Yuan F G 2005 Transient wave propagation of isotropic plates using higher-order plate theory Int. J. Solids Struct. 42 4115-53

[11] Giurgiutiu V 2003 Lamb wave generation with piezoelectric wafer active sensors for structural health monitoring Proc. SPIE 5056 111-22

[12] Cuc A and Giurgiutiu V 2005 Embedded non-destructive evaluation for structural health monitoring, damage detection, and failure prevention Shock Vib. Dig. 37 83-105

[13] Raghavan A and Cesnik C E S 2005 Finite-dimensional piezoelectric transducer modeling for guided wave based structural health monitoring Smart Mater. Struct. 14 1448-61

[14] Raghavan A and Cesnik C E S 2007 3D elasticity-based modeling of anisotropic piezocomposite transducers for guided wave structural health monitoring J. Vib. Acoust. $129739-51$

[15] Raghavan A and Cesnik C E S 2007 Modeling of guided-wave excitation by finite-dimensional piezoelectric transducers in composite plates Proc. 15th AIAA/ASME/AHS Adaptive Structures Conf. (Honolulu, HI)

[16] Lee B C and Staszewski W J 2003 Modelling of Lamb waves for damage detection in metallic structures: part I. Wave propagation Smart Mater. Struct. 12 804-14

[17] Lee B C and Staszewski W J 2003 Modelling of Lamb waves for damage detection in metallic structures: part II. Wave interactions with damage Smart Mater. Struct. 12 815-24
[18] Bent A A and Hagood N W 1995 Anisotropic actuation with piezoelectric fiber composites J. Intell. Mater. Syst. Struct. 6 338-49

[19] Wilkie W K, High J W and Bockman J 2002 Reliability testing of NASA piezocomposite actuators Proc. 8th Int. Conf. on New Actuators (Bremen)

[20] Williams R B, Grimsley B W, Inman D J and Wilkie W K 2004 Manufacturing and cure kinetics modeling for macro fiber composite actuators J. Reinf. Plast. Compos. 23 1741-54

[21] Wilkie W K and High J W 2003 Method of fabricating NASA-standard macro-fiber composite piezoelectric actuators NASA/TM-2003-212427 NASA

[22] Wilcox P, Lowe M and Cawley P 2005 Omnidirectional guided wave inspection of large metallic plate structures using an EMAT array IEEE Trans. Ultrason. Ferroelectr. Freq. Control 52 653-65

[23] Raghavan A and Cesnik C E S 2007 Guided-wave signal processing using chirplet matching pursuits and mode correlation for structural health monitoring Smart Mater. Struct. 16 355-66

[24] Raghavan A and Cesnik C E S 2007 Studies on effects of elevated temperature for guided-wave structural health monitoring Proc. SPIE Symp. on Smart Structures and Materials/NDE 2007; Proc. SPIE $652965290 \mathrm{~A}$

[25] Badcock R A and Birt E A 2001 Use of 0-3 piezocomposite embedded Lamb wave sensors for detection of damage in advanced carbon fibre composites Smart Mater. Struct. 9 291-7

[26] Kessler S S, Spearing S M and Soutis C 2002 Damage detection in composite materials using Lamb wave methods Smart Mater. Struct. 11 269-78

[27] Staszewski W, Lee B C, Mallet A and Scarpa F 2004 Structural health monitoring using scanning laser vibrometry: I. Lamb wave sensing Smart Mater. Struct. 13 251-60

[28] Mallet A, Lee B C, Staszewski W and Scarpa F 2004 Structural health monitoring using scanning laser vibrometry: II. Lamb waves for damage detection Smart Mater. Struct. 13 261-9

[29] Leong W H, Staszewski W J, Lee B C and Scarpa F 2005 Structural health monitoring using scanning laser vibrometry: III. Lamb waves for fatigue crack detection Smart Mater. Struct. 14 1387-95

[30] Staszewski W, Lee B C and Traynor R 2007 Fatigue crack detection in metallic structures with Lamb waves and 3D laser vibrometry Smart Mater. Struct. 18 727-39

[31] Schubert L, Barth M, Klesse T, Kohler B and Frankenstein B 2008 Guided elastic waves and their impact interaction in CFRP structures characterized by 3D laser scanning vibrometry Proc. SPIE $693569350 \mathrm{G}$

[32] Cesnik C E S 2003 Integrated vehicle health management system Proc. NASA Reusable Launch Vehicle URETI (Cleveland, Ohio) NASA Task 5c Annual Report

[33] Salas K I and Cesnik C E S 2008 Design and characterization of the CLoVER transducer Proc. SPIE 6935 69350B

[34] Salas K I and Cesnik C E S 2009 Design and characterization of a variable-length piezocomposite transducer for structural health monitoring J. Intell. Mater. Syst. Struct. at press

[35] Salas K I and Cesnik C E S 2008 CLoVER: an alternative concept for damage interrogation in structural health monitoring systems Aeronaut. J. R. Aeronaut. Soc. at press

[36] Crawley E F and de Luis J 1987 Use of piezoelectric actuators as elements of intelligent structures AIAA J. 25 1373-85

[37] Raghavan A and Cesnik C E S 2004 Modeling of piezoelectric-based Lamb wave generation and sensing for structural health monitoring Proc. SPIE Symp. on Smart Structures and Materials/NDE 2004

[38] Graff K 1991 Wave Motion in Elastic Solids (New York: Dover)

[39] 2007 Engineering Fundamentals Material Database http://www.efunda.com/materials/piezo 
[40] Lim J 1989 Two-Dimensional Signal and Image Processing (Prentice Hall Signal Processing Series) (Englewood Cliffs, NJ: Prentice Hall)

[41] Churchill R V, Brown J W and Verhey R F 1974 Complex Variables and Applications 3rd edn (New York: McGraw-Hill)

[42] Wilcox P D, Lowe M J S and Cawley P 2001 Mode and transducer selection for long range Lamb wave inspection J. Intell. Mater. Syst. Struct. 12 553-65

[43] 2001 ABAQUS/Standard User's Manual Version 6.22001 (Providence, RI: Hibbitt, Karlsson, and Sorensen Inc.)

[44] Werwer A, Polak M A and Santamarina J C 2003 Rayleigh wave propagation for the detection of near surface discontinuities: finite element modeling J. Nondestruct. Evaluat. 22 39-52

[45] Lloyd J M 2004 Electrical properties of macro-fiber composite actuators and sensors MS Thesis Virginia Polytechnic Institute and State University
[46] 2003 Loctite Research, Development and Engineering; Technical Data Sheet for Hysol 9491 http://www.loctite.com

[47] Wilkie W K, Inman D J, Lloyd J M and High J W 2006 Anisotropic laminar piezocomposite actuator incorporating machined PMN-PT single-crystal fibers J. Intell. Mater. Syst. Struct. 17 15-28

[48] Polytec Scanning Laser Vibrometer PSV 400 Hardware Manual 2007 Polytec GmbH

[49] Wilcox P, Lowe M and Cawley P 2000 Lamb and SH wave transducer arrays for the inspection of large areas of thick plates AIP Conf. Proc. Review of Progress in Quantitative Nondestructive Evaluation (Montreal, Canada, 25-30 July 1999); AIP Conf. Proc. 509 1049-56

[50] Salas K I and Cesnik C E S 2009 Guided-wave structural health monitoring in composite plates using CLoVER transducers Proc. 17th AIAA/ASME/AHS Adaptive Structures (Palm Springs, $C A$ ) 